REVIEW ARTICLE

\title{
Defect phenomena in superconducting oxides and analogous ceramic oxides
}

\author{
A M Stoneham and L W Smith \\ AEA Industrial Technology, Harwell Laboratory, Oxfordshire OX11 ORA, UK
}

Received 24 August 1990

\begin{abstract}
In this review we discuss defect phenomena in superconducting oxides. We survey those aspects of oxide superconductors which relate them most closely to conventional ceramic oxides, concentrating on processes and behaviour related to defects. We also identify areas of difference between two types of oxide.

Theoretical modelling of conventional oxides has been extremely effective, and we emphasize that some of these modelling tools can be exploited for the superconducting oxides too. In particular, we stress those methods and ideas that provide a framework for understanding behaviour, those that provide a database of good quantitative experiments and those that provide an established and tested approach to quantitative modelling as a guide to prediction, optimization and extrapolation.

Much progress has been made in both theory and experiment, but some problems do remain and these have not been omitted from our discussions. There is potential to exploit past work on defects in oxides, so as to control defect processes and microstructure and hence to enhance performance.
\end{abstract}

\section{Contents}

1. Introduction

2. Relation of superconducting oxides to other oxides

2.1. Uniformity, stability and stoichiometry

2.2. Oxygen ion behaviour

\subsubsection{Site energies}

2.2.2. Oxygen pressures: exchange with the environment

2.2.3. Oxygen reorganization at constant composition

2.2.4. Volume change with oxygen content

2.2.5. Oxygen desorption

2.3. Interatomic forces and electronic structure

2.3.1. Phonon models

2.3.2. The reference ionic crystal

2.3.3. Aspects of the copper charge and of electronic structure 
3. Point defects in Peroyskites and other oxides

3.1. Basic defect species

3.1.1. Vacancy centres

3.1.2. Interstitials and peroxy-like species

3.1.3. Antisite defects

3.2. Defect-like electronic excitations

3.2.1. Polarons and related defects

3.2.2. Relationship to excitons

3.3. Impurity centres

3.3.1. Intentional substitutions

3.3.2. Comparison with normal ionics

3.3.3. Modelling

3.3.4. Substitution at the anion site

3.4. Unintentional and intentional contaminants

4. Surfaces and interfaces

4.1. Free surface

4.2. Other interfaces

4.2.1. Surface phases

4.2.2. Grain boundaries

4.2.3. Twin boundaries

4.2.4. Subgrain boundaries

4.3. Oxide/metal contacts

4.4. Substrates

5. Solid state processes: I. Diffusion

5.1. Mechanism and control

5.2. Diffusion in non-superconducting oxides

5.3. Types of diffusion constant

5.4. Diffusion in $\mathrm{YBa}_{2} \mathrm{Cu}_{3} \mathrm{O}_{7-x}$

5.5. Diffusion in the La compound $\mathrm{La}_{2-x} \mathrm{Sr}_{x} \mathrm{CuO}_{4-y}$

5.6. Further aspects of diffusion

5.6.1. The reactive element effect

5.6.2. Sintering

5.6.3. Enhanced diffusion

6. Solid state processes: II. Radiation damage and amorphization

6.1. General features: types of damage

6.2. Radiation damage in ionic solids

6.3. Amorphization

6.4. Summary of the main features observed

6.4.1. Optical processes

6.4.2. Gamma irradiation

6.4.3. Electron irradiation

6.4.4. Neutron irradiation

6.4.5. Ion beams

7. Summary

References 


\section{Introduction: superconducting oxides as oxides}

The superconducting properties of oxide superconductors are so striking that it is easy to forget how much these oxides resemble conventional ceramic oxides in other respects. This should not be a surprise; after all, the elemental superconductors are conventional metals in many ways, and especially so at the temperatures at which metal forming operations are carried out. Likewise, in the temperature range at which most superconductor preparation operations are carried out, oxide superconductors are strikingly similar to many other ceramics. In the present review, we shall survey those aspects of oxide superconductors which relate them most closely to conventional ceramic oxides. We shall concentrate on processes and behaviour related to defects, including line and planar defects as well as point imperfections. Our aim is to provide some of the links which might be used to exploit for superconducting oxides the ideas, methods and known behaviour of other systems. We believe there is potential to control defect processes and microstructure so as to enhance performance of the new oxides. In our review it would be over-optimistic to expect solutions to the wide range of materials problems; nevertheless, we believe we may provide the basis for some such solutions.

This similarity between oxides which are superconducting and those which are not should not be taken too far, and indeed this review points to differences as well as resemblances. It is clear that most of the important superconducting oxide phases are themselves unstable, readily transforming into other structures, or changing stoichiometry, or disproportionating. Processing is often controlled by diffusion processes, and it is far from clear whether conditions suitable for effective processing (temperatures high enough for rapid diffusion) are consistent with stability of the desired phase: the best phase for performance may be inaccessible by conventional routes. Moreover, this is not just a question of phase stability; other benefits-like lack of segregation or interdiffusion from substrates-can follow from low temperature processing. What about unconventional routes? Here radiation damage is an obvious tool, whether merely ionizing radiation or more substantial ion beams. In semiconductors, recombination-enhanced diffusion is well established as a phenomenon, though usually as a component of undesirable degradation instead of as a preparative approach. Yet one may ask if apparent rises in critical temperature under neutron radiation correspond to new phases generated which could not be obtained by traditional methods.

Conventional oxides have been studied extensively by many tools, and the experimental insights (a convenient source of a wide range of data is Poole et al 1989) have been strengthened enormously by the effectiveness of theoretical modelling. Crystal structure, phase equilibria, defect nature, defect reactions, surface and grain boundary behaviour, spectroscopy, electronic structure, polaron phenomena, diffusion, and many other features have been predicted with accuracy. Can the same tools be used for superconductors? It is certainly true that several of the proposed phase diagrams for oxide superconductors have adjacent superconducting and semiconducting regions, and one might hope there would be some continuity of properties. Happily, many similar modelling tools can be used, though there are special complications because these oxides are metallic (sometimes only poor metals) even above the critical temperature, and the screening of Coulomb interionic interactions cannot be ignored; the anisotropy of many of the important superconducting oxides adds further complexity. The metallic nature and anisotropy are not only a complication for theory, but also raise difficulty in many experiments, notably in interpreting spectroscopic data. 
Further experimental problems come from the polygranular nature of many samples. On the one hand, both superconducting performance and spectroscopic measurements will be sensitive to alterations of near surface regions (the traditional difficulties of segregation and atmospheric attack); on the other hand, transport measurements may mislead if several phases are present. This effect on transport can be shown in illuminating experiments on gold/oxide superconductor mixtures. Here the composition can be varied continuously, and two different percolation thresholds sought. At higher temperatures, continuous conduction through the more-conducting gold is observed for above $23 \%$ gold; below $T_{\mathrm{c}}$ continuous conduction through superconductor is seen for concentrations above $40 \%$ oxide (Xiao et al 1988). Effective medium theories (Choy and Stoneham 1990) show how both differences in grain type and variations in grain boundary properties affect the way the resistivity and susceptibility change near the critical temperature. Of course, such variations in the grain boundary properties affect normal behaviour as well as superconductivity, as is well known from conventional oxides and exploited in special ways for devices (varistors; negative temperature coefficient of resistance, etc), and indeed scanning tunnelling microscopy (STEM) (Kent et al 1989) indicates insulating regions at boundaries. We describe later the major effects radiation has on grain boundary structure and properties, and note here the importance of a proper representation of this in any model of radiation effects.

The message is that, in any ceramic superconductor (which will usually contain several phases, including unreacted constituents, or possibly different superconductors not resolved by normal methods) the current above $T_{\mathrm{c}}$ and that below $T_{\mathrm{c}}$ may flow through different components. One should not jump to conclusions about, say, the electron-phonon interaction from measurements only in the non-superconducting case.

There is a wealth of phenomena known for ceramic oxides which has relevance to the exploitation of superconducting oxides. In selecting the scope of our review, we have emphasised those topics which provide one of three main features: a framework for understanding behaviour; a database of good quantitative experiments; and an established and tested approach to quantitative modelling as a guide to prediction, optimization and extrapolation. Clearly there are gaps, e.g. the defect modelling does not usually represent well the magnetic behaviour, and the screening of electrostatic interactions may be approximated. Nevertheless, there can be useful reference calculations which offer potential to handle better interactions of superconductor with substrate or with atmosphere, or to achieve better microstructure. Most of our discussion will be for the 1:2:3 compounds, simply because the data are more complete. Nevertheless, the same principles apply to the Tl Bi-based compounds, where existing knowledge may provide a route to still better superconductors.

\section{Relation of superconducting oxides to conventional oxides}

\subsection{Uniformity, stability and stoichiometry}

Before turning to specific properties, it is helpful to ask whether realistic comparisons can be made between superconducting oxides and conventional oxides. The problem is that there are both laboratory to laboratory differences (even uncontrolled but critical differences from one worker to another) and also perhaps significant inhomogeneities in single samples. These problems, whilst diminishing with improved techniques, are present for most oxides. The inhomogeneity can exist within single crystals (for example, Chen et al (1988) report ordered domains of oxygen vacancies with dimensions 
roughly $2 \mathrm{~nm} \times 20 \mathrm{~nm} \times 2 \mathrm{~nm}$ along the $a, b, c$ directions of $\mathrm{YBa}_{2} \mathrm{Cu}_{3} \mathrm{O}_{7-x}$ (YBCO) crystals) or even within individual grains (for example, Morss et al (1988) note variations in the a dimension on scale of about $100 \mathrm{~nm}$ in single grains of YBCO).

There is thus a question of whether the samples used are uniform. Fiory and Grader (1988) remark that a homogeneous oxygen-defect system is necessary for the universal relationship displayed by temperature-scaled quantities $R_{\mathrm{H}} T$ (the Hall coefficient) and $R / T$ (the resistance) (see figure 2 of their paper). Other workers carefully separate out results for given oxygen concentration, rather than merely quoting a nominal composition and temperature. A good example is the paper by Yamaguchi et al (1988), where the resistivity is plotted as a function of $x$ for $\mathrm{YBa}_{2} \mathrm{Cu}_{3} \mathrm{O}_{7-x}$. Three regions are clearly observed:

$\begin{array}{ll}\text { Region I } & x<0.4 \text { the resistivity rises with } T \\ \text { Region II } & x \approx 0.4 \\ \text { Region III } & x>0.5 \text { the resistivity decreases with } T .\end{array}$

The temperature dependence of the resistivity in regions $\mathrm{I}$ and $\mathrm{II}$ is metallic, increasing as the temperature rises. Region III corresponds to the orthorhombic phase and the behaviour in that region is semiconducting, with the conductivity rising with temperature.

A further complication is that the oxides are not inherently stable in the atmosphere. Solution calorimetry measurements can be used to assess the stability of particular oxides. In this way, Morss et al show that $\mathrm{La}_{2} \mathrm{CuO}_{4}$ and $\mathrm{YBa}_{2} \mathrm{Cu}_{3} \mathrm{O}_{6.93}$ are both unstable against reaction with the normal atmospheric constituents, water and carbon dioxide. Many conventional oxides show similar reactions; we shall discuss later the equilibrium with oxygen. Comparisons of values for YBCO from solution chemistry (e.g. on quenched samples) with other measurements of equilibrium situations all seem to yield partial molar enthalpies of solution of about -190 to $-200 \mathrm{~kJ} \mathrm{~K}-1 \mathrm{~mol}\left(\mathrm{O}_{2}\right)^{-1}$ i.e. 1.97 to $2.07 \mathrm{eV}$ per particle. Oesterreicher and Smith (1987) give a value of $43.4 \mathrm{kcal} \mathrm{mol}\left(\mathrm{O}_{2}\right)^{-1}$ (i.e. $1.88 \mathrm{eV}$ ) from thermogravimetric data (their value for the corresponding entropy is $48.6 \mathrm{cal} \mathrm{K} \mathrm{K}^{-1} \mathrm{~mol}\left(\mathrm{O}_{2}\right)^{-1}$, i.e. $\left.2.107 \mathrm{eV} \mathrm{K}{ }^{-1}\right)$; this value is not strongly dependent on $x$ for $7-x>6.8$, but decreases for $7-x<6.7$. This decrease corresponds to disorder in the oxygen sublattices (note their definition of the signs of the enthalpy and entropy through $\ln p=(h / R T)-(s / R))$.

The problems are not equally severe for all oxide superconductors. In particular, $\mathrm{BiSrCaCuO}$ (BISCCO) has advantages of compositional stability, inertness to moisture, ease of oxidation in air and less reactivity with substrate material. Since BISCCO comprises at least two superconducting phases, departures from a precise stoichiometry are less crucial than for $\mathrm{YBCO}$, which may coexist with insulating or semiconducting phases. Oxygenation also seems to be easier and more stable in BISCCO. Yet even for this system, the product can be sensitive to mode of preparation. Most efforts to prepare BISCCO result in a multi-phase sample with a clear transition at about $85 \mathrm{~K}$ and with evidence of a phase transition at about $110 \mathrm{~K}$. In films of BISCCO produced by laser ablation, the wide compositional changes found depend on the temperature of the substrate during composition (Kolinsky et al 1989). For $T>500^{\circ} \mathrm{C}$ the films are rich in copper and deficient in bismuth; for $T<300^{\circ} \mathrm{C}$ the films were rich in bismuth. The content of calcium and strontium varied very little. The variations in composition radically alter the electrical properties.

The sensitivity of superconducting behaviour to phase is striking in copper-based oxides, and is also evident in other oxide systems. Thus, of the five major phases 
of $(\mathrm{Ba}, \mathrm{K}) \mathrm{BiO}_{3}$, only the cubic perovskite phase shows superconductivity (Pei et al 1990).

\subsection{Oxygen ion behaviour}

Here we note some of the ways in which the new oxide superconductors have specific analogies with several distinct types of oxide ceramics. We may relate them to the perovskites on the basis of their structure, or to other ternary oxides as examples of the many structural rules and the use of the concept of ionic radii. We might draw parallels with zirconia or urania, as examples of non-stoichiometric oxides with significant oxygen disorder and rapid oxygen diffusion, or to the known small polaron conductors, including $\mathrm{La}(\mathrm{Sr}) \mathrm{CrO}_{3}$, for their electrical transport behaviour at higher temperatures. Further properties, like key processing energies (for example the desorption energy (Tu et al 1987)) will be mentioned later. In the present section, we shall concentrate on oxygen ion behaviour, and especially on the energetics of oxygen reorganization both at constant oxygen concentration and when there is exchange with the environment.

2.2.1. Site energies. Among the key issues are oxygen-oxygen interactions and their description at a phenomenological level. The site occupancies for oxygen are controlled by two main energies. One is the energy to insert an oxygen into some average site from the environment (taking due care about whether the reference state is molecular, atomic, or some other form of oxygen). It is this energy which gives the main trend of oxygen pressure with temperature. The other major energy is an oxygen-oxygen interaction energy which affects ordering. Again, one must be careful in relating this to specific theoretical numbers; a discussion of similar issues for hydrides is given by Oates and Stoneham (1983) (note in particular the configuration-independent interactions). There will, of course, be further energies leading to site preference. It is these which lead beyond mean-field theories. Whilst a mean field theory can discuss behaviour in terms of relative proportions of sites occupied, the real structure may show much more short-range order, as in specific proposals for vacancy ordering (Golovashkin et al 1988) which appears to explain the temperature dependence of the orthorhombic lattice constants of YBCO. These were observed to increase slowly with temperature, but have a marked change just below the critical temperature. This peculiar behaviour in the structural parameters suggests the presence of some sort of slow diffusion-type structural relaxation in that temperature range, perhaps (Golovashkin et al 1988) from the ordering of oxygen vacancies (Goodenough 1987, 1990).

2.2.2. Oxygen pressures: exchange with the environment. The physical and chemical properties of the high-temper ature superconductors depend on their oxygen deficiency. For example, as the oxygen deficiency decreases, the critical temperature falls, the electrical resistance shows a semiconducting type behaviour at low temperatures, the magnetization at low temperatures shows the presence of quasi-isolated magnetic ions, and the charge carrier density falls. For BISCCO, where there are far fewer reported studies of the properties of the superconducting phase with different oxygen concentrations, the dependence on oxygen concentration remains unclear. The properties of all three superconducting phases of BISCCO (with $T_{\mathrm{c}} \approx 10 \mathrm{~K}, 80 \mathrm{~K}$ and $110 \mathrm{~K}$ ) would need to be studied as a function of the oxygen environment and, to complicate the situation further, it is not yet possible to get pure samples of the $110 \mathrm{~K}$ phase. The $80 \mathrm{~K}$ phase is most easily obtained, and thus most widely studied; for this phase, the 
increase of critical temperature on quenching may be associated with a loss of oxygen. This may explain the enhancement of $T_{c}$ for this phase by reduction with hydrogen. For the $110 \mathrm{~K}$ phase (Bokhimi et al 1989) it appears that removal of oxygen decreases the critical temperature, as for $\mathrm{YBCO}$ but quite different from the $80 \mathrm{~K}$ BISCCO phase.

There are a number of obvious complications which arise for non-stoichiometric oxides with several inequivalent oxygen sites, whether these are superconductors or not. The first is that chemical composition does not give a complete description of the state of the oxide. Even simple properties, like the lattice parameters, will be determined by the numbers of oxygens on specific sites, so that it is important to know whether or not equilibrium over the sites is achieved. The second is that, because the unit cell size varies with oxygen concentration, the total applied pressure, and not just the oxygen partial pressure, will together determine equilibrium. If hydrostatic pressure is applied, the equilibrium will favour more oxygen, as adding oxygen decreases the cell size. So, in experiments to examine the effects of hydrostatic pressure, has one established whether the effects of pressure are really at constant oxygen, or do they show the effects of a new oxygen equilibrium?

\subsubsection{Oxygen reorganization at constant composition. The redistribution of oxygen} over inequivalent sites in YBCO has attracted enormous attention, for it relates directly to the tetragonal/orthorhombic transition. The orthorhombic phase appears to be essential for superconductivity; a few cases are found (especially with doping) where the tetragonal phase coexists with superconductivity, but it is possible that disproportionation into several component oxides has occurred. The oxygen ordering can be described as vacancy ordering within a particular sublattice. This may be more helpful in relating the types of defect structure to those seen in other non-stoichiometric oxides like $\mathrm{FeO}$.

Clearly it is important to have estimates of oxygen ordering energies, and it proves that the energies fitted, usually within mean field theories or some comparable parametrizations, are very similar to those predicted in other systems. Related to this, one may ask is how the results compare with energies expected from a simple elastic continuum model, and indeed the values are in broad accord.

One helpful analysis (Bakker et al 1987) assumes that the oxygen disorder for YBCO is only in the $\mathrm{CuO}$ planes, and that there is equilibrium both between the solid and gas phases and between sublattices. Minimising the free energy (with the configurational entropy included) and, using an open system order-disorder model, leads to two basic parameters. The first is the site energy of $-3.71 \mathrm{eV}$, this being the energy to remove an atom from the gas and place it in position in the solid; it differs from the heat of solution (which thus has the value $-1.17 \mathrm{eV}$ ) by half the oxygen molecular dissociation energy. The second energy represents oxygen-oxygen repulsion, and has the value $0.17 \mathrm{eV}$, in line with values for many other oxides or hydrides. The use of other experimental data yields $0.218 \mathrm{eV}$ for repulsion and $-3.62 \mathrm{eV}$ for the site energy. There is an interesting consequence with either set of parameters, for the model predicts an ideal $\mathrm{YBa}_{2} \mathrm{Cu}_{3} \mathrm{O}_{8}$ (eight, rather than just seven oxygens) (i.e. the oxygen content of the $\mathrm{CuO}$ planes is predicted to begin to rise toward a stoichiometry of $\mathrm{CuO}_{2}$ ) since the site energy is sufficiently low to overcome the oxygen-oxygen repulsions. The configuration-independent interaction noted above (Oates and Stoneham 1983) is not considered in any of the treatments discussed here.

Broadly similar conclusions come from four different analyses by other workers. In 
the first approach, the observed YBCO oxygen pressure $p(T)$ may be analysed using a Bragg-Williams model (Strobel et al 1987) to give a value of about $0.077 \mathrm{eV}$ for the repulsive interaction between oxygens on the $\mathrm{O}_{4}, \mathrm{O}_{5}$ sites; the same data lead to a binding energy of $0.77 \mathrm{eV}$ for an isolated oxygen atom. The equilibrium $\log [p]$ curves are linear in $1 / T$ and yield an activation energy of 1.5-1.65 eV. A second analysis using the Lacher model (Burger et al 1987) determined the enthalpy of formation of oxygen excess $x$ (defined for $\mathrm{YBa}_{2} \mathrm{Cu}_{3} \mathrm{O}_{6+x}$ ) as $-0.7 \mathrm{eV}$ per atom and the repulsive oxygen-oxygen interaction energy as $0.11 \mathrm{eV}$. Other workers examined the dependence of oxygen content on oxygen pressure. The third approach (Monod et al 1987) implied a value for the repulsive interaction between oxygen sites of $0.60 \pm 0.05 \mathrm{eV}$, larger than obtained from $p(T)$, and out of line with other data. In the final example, the kinetics and thermodynamics of the reaction of molecular oxygen with $\mathrm{YBa}_{2} \mathrm{Cu}_{3} \mathrm{O}_{7-x}$ gave further values (Farneth 1988). From experiments at oxygen pressures of $0.05,0.21$ and 1 atm the reaction enthalpy was $\mathrm{d} H=15.1 \mathrm{kcal} \mathrm{mol}^{-1}$ (i.e. $64.39 \mathrm{~kJ} \mathrm{~mol}{ }^{-1}$ or $0.655 \mathrm{eV} /$ particle) and the reaction entropy $\mathrm{d} S=13.9 \mathrm{cal} \mathrm{mol}^{-1} \mathrm{~K}^{-1}$ (i.e. $59.28 \mathrm{~J} \mathrm{~mol}^{-1} \mathrm{~K}^{-1}$ ) for $x=0.3$; for $x=0.6$, there are slight changes. Detailed oxygen evolution experiments on YBCO for $x=0.15$ give results in good agreement with neutron diffraction experiments (Strauven et al 1988). Analysing them using desorption relations yields activation energies of the different desorption processes. In particular, the oxygen-oxygen repulsion energy responsible for the order-disorder transition was estimated to be $(0.16 \pm 0.4) \mathrm{eV}$. (The value of $0.6 \mathrm{eV}$ obtained by Monod et al (1987) is significantly at variance with this). Using the same data with a theoretical model of the phase transition due to Bakker et al and a fit to experimental x-ray diffraction data, Schuller (Schuller et al 1987) gives $1.2 \mathrm{eV}$ for the heat of solution per atom and an oxygen-oxygen repulsion energy of $0.22 \mathrm{eV}$. Strauven's experimental data thus supports Bakker's model rather than Monod's, and the clear overall picture indicates energies similar to those known for other non-stoichiometric systems.

Most data are available for $\mathrm{YBCO}$, though $\mathrm{NdBCO}$ and $\mathrm{YBCO}$ provide a useful contrast. For both (Shaked et al 1990) the orthorhombic/tetragonal transition seems to define the limit of superconducting behaviour. However, the oxygen concentration for which the transition occurs is different for $\mathrm{NdBCO}$ (6.55 oxygen per formula unit) than for YBCO (6.35 oxygen per formula unit). This implies a reduced $O(1)-O(5)$ repulsion in $\mathrm{NdBCO}$ and appears to be linked to the differences in ionic radii.

2.2.4. Volume change with oxygen content. The oxygen stoichiometry affects the lattice parameters of the crystal. One part of this is evident as the orthorhombic distortion. However, there is also a change in cell volume as oxygen content changes. Contrary to many other systems (for example, hydrogen in metals), the cell expands as oxygen is removed. The change is largest for the orthorhombic phase. There are several reasons why this might happen. First, the oxygen affects the Fermi level, and the cell volume will depend on that. If this were the main effect, then similar changes could be achieved by doping, though we know of no checks. Secondly, there are effects which depend on anharmonicity of the host lattice; here there are analogies with simple ionic crystals, where the signs of volume changes were previously a source of controversy.

There is clearly a relation between the volume change with oxygen content and the hydrostatic pressure dependence of superconducting properties. One must always be certain whether behaviour has been studied at constant oxygen content or at constant external oxygen pressure, since these are not the same. If oxygen equilibrates, 
then there will more oxygen at the higher pressure, with a corresponding increase in critical temperature. The extent to which this matters will depend on whether a grain boundary or a bulk effect is dominant.

One useful result can be obtained from lattice parameter data (Tarascon et al 19,87, Salomons et al 1987) for YBCO and similar 1:2:3 compounds prepared under various oxygen pressures and analysed thermogravimetrically. The lattice dilation varies as a function of oxygen pressure: oxygen contracts the lattice strongly in the orthorhombic structure, but has little effect in the tetragonal phase. Partial molar volumes can be deduced from $\mathrm{d} L / L$, the isothermal relative length change of $\mathrm{YBa}_{2} \mathrm{Cu}_{3} \mathrm{O}_{x}$ as a function of oxygen pressure. For the tetragonal phase $\mathrm{d}[\mathrm{d} L / L] / \mathrm{d} x=-0.0009$, and hence its partial molar volume of oxygen is $-0.29 \mathrm{~cm}^{3} \mathrm{~mol} \mathrm{O}-1$. For the orthorhombic phase $\mathrm{d}[\mathrm{d} L / L] / \mathrm{d} x=-0.017$ and hence its partial molar volume of oxygen is $-3.8 \mathrm{~cm}^{3} \mathrm{~mol} \mathrm{O}^{-1}$, an order of magnitude larger.

The lattice parameters do, of course, give more information about the deformation associated with particular site occupancies. Thus the temperature dependence of oxygen site occupancy at a constant oxygen pressure may be analysed to indicate the concentration dependence of the axial lengths $a$ and $b$ at room temperature; theoretical values (Nakamura et al 1988) compare very well with experiment.

2.2.5. Oxygen desorption. One key question for processing concerns which are the sites from which oxygens come off in desorption and which gain oxygen when oxygen is added. There is, of course, a natural asymmetry between absorption and desorption; there is a further asymmetry because any subsequent diffusion will depend on the stoichiometry of the solid. Ion beam effects will be discussed later.

Two interesting results relate to the sites from which desorption occurs. First, the temperature at which oxygen desorbs seems to depend on whether there are chain sites present or not. Chain sites make desorption easier, and their presence or absence suggests why activation energies for desorption are higher in some cases (like the $\mathrm{Bi}$ compounds) than others. Oxygen evolution experiments in La and YBCO ceramic superconductors (Loquet et al 1988) show that oxygen desorbs at $600^{\circ} \mathrm{C}$ from $\mathrm{Cu}-\mathrm{O}$ chains (when present) with an activation energy of $1.2 \mathrm{eV}$, whereas the desorption from other oxygen-containing planes occurs at $800^{\circ} \mathrm{C}$ with a higher activation energy of $2.8 \mathrm{eV}$. Similar studies (Hegde 1988) obtain an activation energy for oxygen desorption from superconducting (orthorhombic) YBCO to be $28 \mathrm{kcal} \mathrm{mol}^{-1}$ (i.e. $1.2 \mathrm{eV}$ ) and from non-superconducting (tetragonal) YBCO to be $54 \mathrm{kcal} \mathrm{mol}^{-1}$ (i.e. $2.35 \mathrm{eV}$ ). The study shows clearly that the oxygen desorbing below $470^{\circ} \mathrm{C}$ can be assigned to the loosely bound bridging oxygen in the $\mathrm{Cu}-\mathrm{O}$ chain along the $b$ axis. Further, only two types of oxygen are detected in the desorption study, and these can be correlated with the two distinct $\mathrm{Cu}-\mathrm{O}$ distances in the 1:2:3 structure. This view is further supported by the near absence of the chain oxygen in the non-superconducting phase and the absence of oxygen desorption below $470^{\circ} \mathrm{C}$ in a nitrogen-heated sample.

However, there is a second observation (Marwick et al 1988) that oxygen does not outgas especially from the chain sites under ion beam bombardment, so that losses from these chain sites are not the only factor to consider in explaining the resistivity changes of a factor of $10^{4}$ on irradiation of YBCO by $2 \mathrm{MeV}$ arsenic ions.

It is well known that moisture affects observations on conventional ceramic oxides; this is, after all, the basis for some moisture sensors. Not surprisingly, many workers have investigated the effects of water and atmosphere on ceramic superconductors. It is not always clear whether the effects seen are principally surface, or grain boundary, or 
bulk phenomena. Certainly some observations on $\mathrm{YBCO}$ are consistent with changes in the extent of oxidation. Saiz and Moya (Saiz et al 1988) found that, on exposure to air, the oxygen mass gain varies linearly with time and is dependent on both crystal structure and density. Tetragonal samples were found to oxidize about 50 times faster than orthorhombic ones. Harris and Nyang (1988) looked at the loss of superconductivity caused by exposure to moist air. (This is discussed in more detail in the section on surface phases). Other work (Horowitz et al 1988) shows that reaction to moisture is rapid for the first 48 hours, then quite slow. The sinterability and superconductivity follows a similar pattern of deterioration, suggesting surface reaction products cause the property degradation. It is unfortunate that beneficial changes in electrical behaviour with stoichiometry (Regnier et al 1988) seem to correlate with significantly poorer resistance to ageing and humidity. In contrast, BISCCO is inert to moisture: the superconducting properties of BISCCO films on $\mathrm{MgO}$ substrates do not seem to be significantly influenced by the presence of water vapour during the different steps of fabrication (Fogarassy et al 1988).

\subsection{Interatomic forces and electronic structure}

In conventional oxides, elastic and lattice vibrational data are the key to understanding interatomic forces, and these in turn have provided a basis for modelling a wide range of defect phenomena, including the properties of polarons (see Catlow and Mackrodt (1982) and the special issue of J. Chem. Soc. Faraday Trans. II (1989) on the MottLittleton method). The central issue to examine here is whether phonon spectra (infra-red, Raman) and elastic properties do really say anything useful about lattice dynamics and defect behaviour in oxide superconductors, and especially about changes at the critical temperature. Fracture and crack propagation are more complex, but it is the character of these same interatomic forces which influences mechanical properties, and notably the brittleness of these oxides.

2.3.1. Phonon models. In considering phonon spectra, the questions we might ask are: Are the derived force constants like those for non-superconducting oxides? Why might this be-do the features which give rise to superconductivity really have no major influence on interatomic forces?

The dominant terms in the energies which lead to interatomic forces and cohesion of non-metallic oxides are (i) Coulomb interactions, (ii) short-range repulsion, and (iii) polarization-related terms. The short-range repulsions are the terms which underly the concept of ionic radii, and so have wider approximate validity. The unscreened Coulomb interaction dominates the total energy (effectively the cohesive energy, but, strictly speaking, the energy to take the crystal into its component ions) but is far less important in the force constants which determine phonon frequencies (Hayes and Stoneham 1985, p 58). The implication is that, for defect energies, if the extent of screening is uncertain, we may retain some confidence in those cases which do not alter the main Coulomb part (for example, substitution of one ion by another of the same charge) but should have reservations about cases where the net charge (or the number of ions) changes.

In $\mathrm{La}_{2} \mathrm{CuO}_{4}$ the infra red spectra are essentially those of insulators, so that the infrared active phonons are easily identified. After doping with $\mathrm{Sr}$, $(\mathrm{Sr} 0.08$ per formula unit) the electronic contribution to the $\mathrm{a}-\mathrm{b}$ conductivity tends to screen the in-plane phonons, but the out of plane response is largely unaltered. This implies a very large conductivity anisotropy (greater than 50:1) (Collins et al 1989). 
2.3.2. The reference ionic crystal. Most of the oxides which resemble the superconductors are themselves insulators or semiconductors. This can be confirmed by either a look through a structural inorganic chemistry text (for example Wells 1987) or by asking any who have made a large number of such compounds. Moreover, some of the key oxide superconductors show characteristic non-metal features for some range of temperature or composition, or even for some component of the structure (e.g. the $\mathrm{BiO}$ plane in BISCCO (Tanaka et al 1989). These features include conductivity in the normal state which rises with temperature, or even variable range hopping, which implies some sort of a gap. This suggests it should be fruitful to compare oxide superconductors with the better understood ionic oxides, especially because it is these ionic oxides which are especially well understood as regards aspects affecting processing, e.g. diffusion, grain boundaries, shear and twin planes, dopant sites and thermodynamic energies.

One place to start is with systematic calculations for the key superconducting oxides as if they were ionic insulators, since one can then separate special features (perhaps related to superconductivity) from those which are simpler in origin. We begin by looking at the structure-related terms in the total energy, mentioned above:

\section{Real superconductor}

Short-range repulsion (i).

Substantial binding by terms which do not depend much on configuration; (electron gas).

Terms depending on the ionic species and the way they influence the electron gas and its response to the perturbation locally.

Special terms, are often added in for example Jahn-Teller or magnetic or crystal field terms.

\section{Reference ionic crystal}

Short-range repulsion (i).

Substantial binding by Coulomb terms which do not depend much on configuration provided anions and cations coordinate each other (ii).

Polarization terms. These are not independent of repulsion hence the need for a shell model (iii).

Special terms can be added in. Is Peierls distortion a problem?

(i) As represented in the use of ionic radii. However care is needed, because, for example, real crystal structures optimize a free energy and not nuclear spacings, and even repulsive energies (not just radii) depend on coordination (Shannon et al 1969).

(ii) There is a tendency to keep species on specific sublattices. Note also that there is a longitudinal optical-transverse optical splitting for phonons, with accompanying effects on the selection rules (Hayes and Stoneham 1985).

(iii) The issue of screening has been noted above for $\mathrm{La}_{2} \mathrm{CuO}_{4}$.

Short-range repulsion. It is tempting to use arguments based on ionic radii alone to suggest crystal structures, dopant sites and the like. However, the reality is that these are limited guides only, and that systematic studies show strong systematic dependences of radius on coordination number (Goldschmidt et al 1926). Whilst one can put in this coordination dependence, it makes far more sense to use models which are correctly based on free energies, e.g. approaches which use interatomic potentials plus some algorithm for the total energy as a function of ion positions. 
There is a widely used empirical set of 'effective' ion radii using 1000 experimental atomic distances (Shannon et al 1969) and an approximately linear relation between ionic volume and unit cell volume for over 60 isotypic series of oxides and fluorides (see their figure 1). Their figure 2 shows the effective radius as a function of coordination number. Certain effects such as anion-anion repulsion, irregular coordination and metal-metal bonding cause deviations from predicted interatomic distances and are difficult to include in any simple table of radii. The radii show some interesting features, notably the way the rare-earth radii are observed to fall with increasing number of $4 \mathrm{f}$ electrons. The radius of $\mathrm{Y}$ falls between that of $\mathrm{Dy}$ (dysprosium) and that of Hf (hafnium), and this allows a simple relationship between YBCO and the structures of similar rare-earth compounds.

\subsection{Aspects of the copper charge and of electronic structure}

Even if the origin of superconduction is not our present subject, we are interested in knowing about the nature of the carriers: whether they are on the cations or the oxygens or some combination of the two, how this compares with other Mott insulating systems, and what the effects of high carrier concentrations are.

The sites of the carriers is not always obvious, even in relatively simple oxides. In $\mathrm{Li}$-doped $\mathrm{NiO}$, for instance, oxygen $k$-edge $\mathrm{x}$-ray absorption spectra (Kuiper et al 1989, deGroot et al 1989) show that the holes compensating the Li impurity charge are located primarily in the oxygen $2 \mathrm{~d}$ states, rather than in the conventionally assumed $\mathrm{Ni} 3 \mathrm{~d}$ states. These holes are bound to the $\mathrm{Li}$ impurities, forming states in the NiO band gap. The $\mathrm{O} 2 \mathrm{~d}$ holes appear to have a very large antiferromagnetic exchange interaction with neighbouring $\mathrm{Ni}^{2+}$ spins, which makes them appear like low-spin $\mathrm{Ni}^{3+}$ states in macroscopic magnetic measurements.

There are obviously possible similarities between $\mathrm{NiO}: \mathrm{Li}$ and the copper-based oxide superconductors. These altered charge states lead directly to small polaron descriptions: an impurity in a particular net charge state can be described by that charge state or by declaring it to have some standard charge state and to have trapped one or more polarons (see later; clearly the usefulness of one or other description depends on the details of the case (Hayes and Stoneham 1985). The real complication for copper- based oxide superconductors stems from the similarity of the energies of holes on both oxygen and copper, so that a description like $\mathrm{Cu}^{3+}$ can be a useful shorthand without describing all key features well.

Many authors have argued for particular charge assignments for $\mathrm{Cu}$ in the La and $1: 2: 3$ superconductors; to a lesser extent, this has also been attempted for the $\mathrm{Tl}$ and $\mathrm{Bi}$ compounds (Rigney et al 1989). These assigned charges are not necessarily the same as those appropriate in a 'reference crystal' calculation (Catlow and Stoneham 1983). As an example (Steiner et al 1988), an average $\mathrm{Cu}$ charge on the Cu sites in YBCO can be obtained as a function of oxygen concentration from XPS core level spectroscopy. This suggested that the $3+$ and + states were present, in addition to the dominant $\mathrm{Cu}^{2+}$ ions; actual concentrations depend on the annealing conditions of the samples and vary reversibly with the $O$ defect concentration. The implication was that there are holes on the oxygen sites in the basal plane of the crystal structure. The probability for holes on these oxygen sites is about 0.64 and is constant in the semiconducting regime of $\mathrm{x}$ between 0 and 0.3 but decreases to 0 , for $x=\frac{1}{2}$. Such results can be used to relate critical temperature to carrier concentration, which provides a test of a number of theories. They can also be used to construct an energy level diagram for $\mathrm{Cu}^{2+}$ and $\mathrm{Cu}^{3+}$ in $\mathrm{YBCO}$; the $3+$ state seems barely stable. 
The question of which charge states of a multivalent ion are stable in a particular host contains several substantial terms. In semiconductors, hybridization is central; in ionics, the balance between polarization and ionization potential is dominant (Hayes and Stoneham 1985). One striking result suggesting the importance of ionization potentials for oxde superconductors concerns rare-earth dopants in 1:2:3 compounds. Those rare earths which readily go to $4+$ are effective at suppressing superconduction. The free ion ionization potentials (i.e. $3+$ state to $4+$ state) of the free rare-earth ions are as follows $(\mathrm{eV})$ :

\begin{tabular}{|c|c|c|c|c|c|c|c|c|c|}
\hline $\begin{array}{l}\text { Behaviour: } \\
\text { Ionization } \\
\text { potential }\end{array}$ & \multicolumn{7}{|c|}{ Easy to form $4+$} & \multicolumn{2}{|c|}{ Hard to form $4+$} \\
\hline \multirow{2}{*}{$\begin{array}{l}\text { Ionization } \\
\text { potential }\end{array}$} & $<37$ & 37 & 38 & 39 & 40 & 41 & 42 & 43 & 44 \\
\hline & $\begin{array}{l}\mathrm{UCe} \\
\mathrm{Hf}\end{array}$ & & $\operatorname{Pr}$ & $\mathrm{Tb}$ & $\mathrm{Nd}$ & $\begin{array}{l}\text { Sm } \\
\text { Dy } \\
\text { Pm }\end{array}$ & $\begin{array}{l}\text { Ho } \\
\text { Tm } \\
\text { Er } \\
\mathrm{Eu}\end{array}$ & $\mathrm{Yb}$ & $\begin{array}{l}\text { Lu } \\
\text { Gd }\end{array}$ \\
\hline
\end{tabular}

It is tempting to say that the suppression of superconductivity is simply based on $4+$ ion formation. That is simplistic: not only is superconductivity seen at dopant concentrations too large for this (unless there is $\mathrm{Pr} / \mathrm{Y}$ disproportionation) but also detailed studies (Lopez-Morales et al 1990) indicate more subtle behaviour. The doping of YBCO with Pr appears to have its effect through influencing the carrier concentrations in the chains and through its influence on oxygen vacancies. The $\mathrm{Pr}$ appears to remain essentially $3+$, but with a strong $4 f$-oxygen $2 \mathrm{~d}$ overlap. Quite how the trends with ionization potential happen, therefore, is still incompletely understood.

A second significant component is the Madelung energy. As normally used, this is an idea from ionic crystals (though, of course, even the energy of a metal may be partitioned using the same concept). In ionic crystals the Madelung energy has several functions: it is dominant in the cohesive energy; it introduces special features into phonon spectra (like the longitudinal/transverse optic splitting); it keeps ions on a specific sublattice, and favours structures in which ions of one sign of charge are surrounded by ones of the opposite charge. If an oxide superconductor is ionic in some regime (or even in some region, like a grain boundary phase) during processing, the implications of the Madelung potential are the same as in conventional oxides. However, for oxide superconductors, the Madelung potential (or, strictly the value one would find for the difference between that at the $\mathrm{Cu}$ and $\mathrm{O}$ sites in the $\mathrm{CuO}_{2}$ planes in an ionic model) is used to order a series of related crystals (see, for example, Torrance and Metzger 1989). Which oxides are superconducting, which of those that are superconductors will have the highest critical temperature, etc, are the sorts of issues discussed using the Madelung potential as a classifier. The potential, in effect, represents some important features of the structure in a convenient way, and the superconducting behaviour has a common origin with the Madelung potential in these features. The 'reference crystal' approach builds on this systematically.

We can express the paradox in a different way. In III-V compounds, transition metal ions can exist in a range of charge states. For them, there is charge transfer between the anion and cation sublattices, and this hybridization is the route by which the energies of the several charge states can be reduced from many $\mathrm{eV}$ for free ions to less than the band gap. A corollary, however, is that the same mechanism makes antisite disorder (group III atoms on group $V$ sites and vice versa) favourable, and 
such defects are observed and of immense importance. In the ionic (six-fold coordinated) simple oxides, like $\mathrm{MgO}$, where again a range of transition metal ion charge states is seen, the mechanism is wholly different, for hybridization is demonstrably negligible. Instead, ionic polarization is a major component. Moreover, the result of the differences in dominant energies is also the almost complete absence of antisite defects (cations can disorder onto other cation sites, but do not do so to anion sites). When we look at the superconductors, we see arguments for hybridization (like III-V) but no evidence for antisites (indeed, quite the contrary; compare our discussion of radiation damage).

\section{Point defects in perovskite and other oxides}

Most studies of point defects in conventional oxides have concentrated on those in the bulk. This emphasis is not complete, however, for there are substantial and significant studies of surface defects, plus some analogous work on oxide-oxide interfaces. In the new superconductors, there will surely be similar bulk, surface and grain boundary defects. The relative instability of these oxides will lead to altered surface layers, and it is likely that segregation and interaction with the atmosphere will give rise to a wealth of imperfections, whether impurity or defect. Moreover, the defects in superconductors will have special roles. Those at interfaces may limit the intergranular current; those within grains may provide pinning to improve performance. There have been suggestions that point defects are an active component of carrier pairing; this role is harder to accept as a major contributor to superconductivity, since such pairing would need to occur without trapping and carrier localization.

Defects in conventional oxides have been identified in may ways, notably by spectroscopy: optical absorption, luminescence, spin resonance, nuclear magnetic resonance (including quadrupole resonance) and phonon spectroscopy and internal friction. In addition there are studies of defect motion and electronic transport, including such forms as variable-range hopping and polaron behaviour with its activated mobility. Since the oxide superconductors are metals, the tools are somewhat different: optical methods can be of less value, and even electron spin resonance is of limited value, mainly because it appears to come principally from minor phases or other atypical regions. The systematic use of irradiation is another important tool for understanding intrinsic defects. This will be analysed separately, when phenomena discussed include amorphization, oxygen loss, possible sublattice mixing, and defect generation.

\subsection{Basic defect species}

The most complete studies of defects in ternary oxides are probably the systematic studies of perovskites, both from experimental work on strontium titanate (Arizmendi et al 1984) and the specific theoretical studies of barium titanate (Lewis 1983). Theory and experiment together are a powerful combination. As an example, an important prediction from modelling barium titanate is that, whereas pure barium titanate has vacancies of all species in comparable concentrations, the extrinsic (impurity induced) disorder is mainly associated with barium and oxygen defects. 
3.1.1. Vacancy centres. In normal ceramic oxides, vacancies are detected by atomic transport and by optical spectroscopy. Since these oxides are insulators, there are important differences between the alternative charge states. In many cases transport is dominated by vacancies without carriers: in magnesium oxide, for instance, the $\mathrm{Mg}$ ions diffuse by a vacancy mechanism involving doubly charged $\mathrm{Mg}$ vacancies; in barium titanate, the vacancies formed by removing $\mathrm{Ba}^{2+}, \mathrm{Ti}^{4+}$ or $\mathrm{O}^{2-}$ are all present, and contribute to diffusion. When there are seyeral species diffusing, it is the slowest-moving which determines creep and sintering behaviour. The optical and spin resonance spectra are observed for vacancies with trapped carriers. Many simple oxides (i.e. those which involve only closed-shell ions of single dominant valence) show vacancy centres of these types (Bridges et al 1990):

$\mathrm{F}^{+}$centre: An oxygen vacancy in which an electron is trapped. These are observed in spin resonance as well as optically. They are found in $\mathrm{MgO} ; \mathrm{CaO} ; \mathrm{SrO} ; \mathrm{BaO}$; alumina; $\alpha$-alumina; $\mathrm{MgAl}_{2} \mathrm{O}_{4}$; lithium niobate and lithium tantalate.

$\mathrm{F}^{0}$ centre: An oxygen vacancy in which two electrons are trapped. Sometimes it is called the $F^{\prime}$ centre (or even, unhelpfully, $F$ centre). This has been seen in almost all oxides for which the $\mathrm{F}^{+}$centre is seen.

$\mathrm{V}^{-}$centre: A divalent cation vacancy at which a hole is trapped. The hole is often on an oxygen (so the vacancy has a single $\mathrm{O}^{-}$neighbour, instead of just oxide ions);

$V^{0}$ centre: $A$ divalent cation vacancy at which two holes are trapped, normally on adjacent oxygens (Henderson 1988).

There are, of course, cation vacancies involving higher-valent cations, and these are usually known as $V$ centres too. In oxides which have cations which show several charge states, the holes may be on the cation sublattice. In $\mathrm{FeO}$, for instance, the holes would normally be on the Fe. The same has generally been assumed true for $\mathrm{NiO}$, though recent work (deGroot et al 1989) suggests that, for substantial Li doping, the holes move onto the oxygen sublattice. Observations of vacancy-impurity pairs are very common for perovskites and other normal oxides, and will be discussed later; here the vacancies provide charge compensation.

Whilst there are direct observations of vacancies in superconducting oxides by high resolution electron microscopy (at $0, \frac{1}{2}, 0$ sites (Liang et al 1989)), most information is based on transport, on vibrational spectra, or on the various core spectroscopies. In the La compounds there is a strong dependence of diffusion coefficient on stoichiometry (diffusion itself is discussed later), and this can be understood by simple assumptions about the role of $\mathrm{Cu}^{3+}$ ions. The concept of localized charges with mobile carriers needs care; in many cases the charge state is merely a shorthand for some more general structural feature. This is a special problem arises which pervades much defect work for oxide superconductors: in what sense should one believe in $\mathrm{Cu}^{3+}$ ?

Core spectra. It was suggested at a relatively early stage (Steiner et al 1987) that the XPS and UPS spectra of the La compounds indicated oxygen vacancies. Later observations (Werfel et al 1988) suggested that oxygen vacancies could explain the two Ba configurations observed by XPS core-level data.

Core spectroscopies suggest the holes are on the oxygen sublattice, rather than the $\mathrm{Cu}$ sublattice, though there are plenty of claims for the $3+$ state of $\mathrm{Cu}$. In the present work we shall adopt the working hypothesis that for most purposes it is not necessary to identify whether the holes are on $\mathrm{Cu}$ or $\mathrm{O}$. Obviously in checking charge compensation, or in relating one member of a family of oxides to another, it is useful 
to adopt an assumption of $\mathrm{Cu}^{3+}$, but this need be little more than an accounting procedure. The problem is very much the same as in covalent solids, where quite distinct descriptions can be used provided they are followed systematically (Catlow et al 1988). For example, one may wish to exploit similarities with non-superconducting oxides. Thus, for instance, $\mathrm{La}_{2} \mathrm{NiO}_{4}$ appears to contain $\mathrm{La}$ vacancies compensated both by holes and by oxygen vacancies (Choisnet et al 1983), and one might wish to use a similar description for metallic oxides with distinct sublattices.

Local charge balance arguments in relation to the site which the $\mathrm{Cu}^{3+}$ might occupy and to the stoichiometry dependence of diffusion lead to the following conclusions:

(i) the strong dependence of the diffusion constant, $D$, on the oxygen vacancy concentration for La oxides is not expected for YBCO;

(ii) there will be two-dimensional oxygen diffusion in YBCO, whereas it is effectively one-dimensional for the La oxides;

(iii) the $\mathrm{O} 1$ and $\mathrm{O} 4$ oxygens in the 1:2:3 compounds (i.e. three out of the seven oxygens) should be especially mobile (Routbort et al 1988).

Vibrational spectra. Many workers have noted the effects of defects on the vibrational spectra of superconducting oxides. The key observations are for $\mathrm{YBCO}$, where the main features are as follows.

(i) A Raman peak at $550-600 \mathrm{~cm}^{-1}$ because the $\mathrm{Cu}(1)-\mathrm{O}(4)$ stretch vibration in the $\mathrm{Cu}-\mathrm{O}$ chains becomes Raman active with increasing oxygen vacancy disorder (Kourouklis et al 1987, Liu et al 1988, Krol et al 1987, Hangyo et al 1988, McCarty et al 1988).

(ii) Minor changes at $390 \mathrm{~cm}^{-1}$ are observed in Raman spectra (Kourouklis et al 1987). For $\mathrm{MBa}_{2} \mathrm{Cu}_{3} \mathrm{O}_{7}$, with $\mathrm{M} \equiv \mathrm{Y}, \mathrm{Eu}$ and $\mathrm{Gd}$, all three systems have very similar Raman features. In oxygen-deficient $\mathrm{YBCO}$ a distinct Raman peak, a distinct peak emerges. The suggestion (Rosen et al 1987) is that that this could correspond to a stretch of the $\mathrm{Cu}(2)-\mathrm{O}(2,3)$ bond. A peak is also reported at $150 \mathrm{~cm}^{-1}$ in infra-red reflectance measurements (Crawford et al 1988). This peak grows with increasing oxygen content. As the additional oxygen goes predominantly into the linear $\mathrm{Cu}-\mathrm{O}$ chains, a natural assignment for this mode is as a linear $\mathrm{Cu}-\mathrm{O}$ chain vibration.

Excitations. Optical excitations associated with defects are of three main types. First there are the straightforward excitations from one electronic state to another; these may often be related to theoretical one-electron levels, at least qualitatively. An example is the $\mathrm{F}$ band in oxides, from the excitation of an electron trapped in an oxygen vacancy. Secondly, there are the transitions known variously as charge transfer, or polaronic, where the lattice deformation associated with localized charge is critical; such transitions do not appear in standard band structure approaches. Here an example is the excitation of trapped holes at cation vacancies in oxides. Thirdly, there are excitations associated with lattice vibrations where the defect alters selection rules or spectral weights; examples were noted earlier. It is not always clear into which category defect spectra in oxide superconductors fall. Indeed, in many cases it is far from clear that particular spectra are intrinsic, rather than impurity related, especially when the impurity may be water or carbon.

In studies of the visible-near-ultraviolet optical spectra of YBCO (Kelly et al 1988) two strong electronic transitions are reported which are activated by oxygen deficiency. The first at $4.1 \mathrm{eV}$ is associated with $\mathrm{Cu}^{1+}$ and is identified with the 
existence of a highly localized $\mathrm{O}-\mathrm{Cu}(1)-\mathrm{O}$ complex. A second excitation is seen at $1.7 \mathrm{eV}$ when there is oxygen deficiency or when cobalt substitution makes the material non-superconducting. This may be a $\mathrm{d}-\mathrm{d}$ transition of $\mathrm{Cu}^{2+}$. Neither transition can be explained in terms of existing band structure calculations. Similar work (Garriga et al 1988) on semiconducting YBCO $(x<0.65)$ shows two rather intense peaks at $1.75 \mathrm{eV}$ and $4.1 \mathrm{eV}$ whose intensities depend strongly on oxygen content, though their energies are hardly dependent on oxygen content or temperature. The excitation at $4.1 \mathrm{eV}$ appears to be associated with excitations involving transitions from $\mathrm{Ba}(5 \mathrm{~d})$ and/or $\mathrm{Cu}(3 \mathrm{~d})$ to $\mathrm{O}(2 \mathrm{p})$ states. The peak at $1.75 \mathrm{eV}$ may be due to $\mathrm{Cu}(3 \mathrm{~d})$ and $\mathrm{O}(2 \mathrm{p})$ states.

3.1.2. Interstitials and peroxy-like species. Interstitials are common in the relatively open structures of some oxides. Examples include the interstitial ions in the fluorite oxides like urania, or molecular oxygen interstitials in the various forms of silica. In the closer-packed oxides, like magnesia, there are occasional suggestions of interstitial molecular ions, like $\mathrm{O}_{2}^{-}$, possibly in association with other defects. In $\mathrm{La}_{2} \mathrm{NiO}_{4+z}$, neutron scattering shows a relatively simple interstitial adjacent to four $\mathrm{La}$ ions (Jorgensen et al 1989).

One interesting transient species is the self-trapped exciton where in one model, in effect, an oxygen atom moves from its normal site to form a peroxy or similar species, leaving a neutral vacancy (or $\mathrm{F}^{0}$ centre). The peroxy species has become a common model for a number of defect signals, and this may be appropriate. However, it must be added that others have suggested that hydroxyl, not peroxy, is responsible for some of the features.

Whilst it is easy to envisage peroxy species in insulating oxides, there is a question of what binding is expected to stabilise the peroxy defect in a metallic oxide? Many authors have interpreted features of core spectroscopies as being due to molecular ions, usually in the $\mathrm{O}_{2}^{2-}$ state. For example, the x-ray photoelectron spectra in the $\mathrm{O}(1 \mathrm{~s})$ region shows (Rao et al 1987) the presence of peroxide-like species with a high binding energy (about $533 \mathrm{eV}$ ) besides the low binding energy peak (about $529 \mathrm{eV}$ ) due to the oxide ion. The proportion of the peroxide-like species increases as the temperature is lowered and this phenomenon is fully reversible. The suggestion is that the peroxidelike species is formed by the oxygens of the $\mathrm{BaO}$ layer by hole-hole coupling. The 2 - charge state of the oxygen molecule has been deduced in $\mathrm{La}_{2} \mathrm{NiO}_{4.6}$ (Buttrey et al 1988) but is not stable in free space; if correctly identified, it must be bound in the metal only indirectly or because somehow the electronic screening of the Coulomb repulsion exceeds the diminution in direct bonding. One idea (Manthiram et al 1988) is that the ordering of one-electron energy levels is such that holes can trap out onto oxygen $2 \mathrm{p}$ orbitals in the peroxy-like species. The resistivity and superconductivity seem to be consistent with this idea.

As the oxygen stoichiometry varies in YBCO there is clearly a question of definition as to whether, say, 6.5 oxygens per formula unit represent one interstitial every two units of a structure with 6 oxygens per cell or one vacancy in a structure with 7 oxygens per cell. However, there is a reported dependence of the way oxygen is incorporated on stoichiometry (Hervieu et al 1987), suggesting intercalation of oxygen as a dimer for $x=6.5-6.9$, and intercalation as monomeric oxygen for $x=6.94-7$. Clearly the excess oxygen for $x>7$ presents different problems; here the suggestion is that the excess is incorporated into the $\mathrm{CuO}_{2}$ layers, but no specific interstitial model is given. With excess oxygen, there remains a question of its charge state and the location of any 
holes should electrons transfer to the oxygen to give an anion. X-ray photoelectron spectra (XPS) and ultra-violet photoelectron (UPS) spectra (Hegde and Ganguly 1988) for oxides of lead and bismuth show that only the $6 \mathrm{p}$ electrons of $\mathrm{Pb}$ and $\mathrm{Bi}$ (and not the $6 \mathrm{~s}$ electrons) are ionized on oxidation of the metal to $\mathrm{PbO}$ or $\mathrm{Bi}_{2} \mathrm{O}_{3}$. The $6 \mathrm{~s}$ band lies below the oxide $2 \mathrm{~d}$ band, so that the metals are not ionized to form $\mathrm{Pb}^{4+}$ and $\mathrm{Bi}^{4+}$ as expected, but remain in $\mathrm{Pb}^{2+}$ or $\mathrm{Bi}^{3+}$ states, and the oxygen forms the lower-valent molecular ion $\mathrm{O}_{2}^{2-}$. Similarly, in a superconducting oxide, the $\mathrm{Cu}^{2+}(3 \mathrm{~d})$ band overlaps completely with the $\mathrm{O}^{2-}(2 \mathrm{p})$ band in $\mathrm{YBa}_{2} \mathrm{Cu}_{3} \mathrm{O}_{6.95}$, and the excess oxygen can be stabilised through lower-valent oxide ions, instead of $\mathrm{Cu}^{3+}$.

For the semiconducting compositions $\mathrm{YBa}_{2} \mathrm{Cu}_{2} \mathrm{CoO}_{7.25}$ and $\mathrm{YBa}_{2} \mathrm{Cu}_{2.5} \mathrm{Fe}_{0.5} \mathrm{O}_{7.19}$ $\mathrm{X}$-ray structural analysis, conductivity and susceptibility measurements, and thermogravimetric data (Tao et al 1988) provide evidence for Co or Fe substitution at the $\mathrm{Cu}(1)$ position and intercalation for peroxide ions.

3.1.3. Antisite defects. Antisite defects in ionic crystals are rare in binary compounds, where the Madelung potential keeps cations off anion sites. There are exceptions, however; radiation damage in alkali halides creates perfect dislocation loops, which means that the initial anion disorder has to be accompanied by subsequent cation disorder. The key in this case appears to be neutral diatomic halogen molecules at anion-cation divacancies. In covalent crystals, like III-V compounds, antisite defects are much more readily formed.

Ternary and more complex oxides have more than one cation sublattice, so one can have one cation on the site appropriate for another. Such defects are formed under irradiation,but can also exist in suitable cases in thermal equilibrium. Schirmer and Muller (Schirmer et al 1973) observed trivalent $\mathrm{Ti}$ on Si sites in strontium titanate after neutron irradiation. In lithium niobate, almost all the disorder appears to be on the cation sublattice. The situation is very different in barium titanate, where the large difference in ionic radii tends to give strong site preferences (Lewis and Catlow 1983).

\subsection{Defect-like electronic excitations}

9.2.1. Polarons and related defects. Here we find inevitable issues of definition, since an impurity in a particular charge state can be described either by that charge state or by declaring it to have some standard charge state and to have trapped a polaron. In an $\mathrm{ABO}_{3}$ system, for example, the polaron might involve a change of charge of $\mathrm{B}^{N+}$ to $\mathrm{B}^{N+1}$ at a $\mathrm{B}$ site. The polaron can be an electron (so that one might have $\left[\mathrm{M}^{5+}+\right.$ $\mathrm{e}^{-}$] described as $\mathrm{M}^{4+}$, with $\mathrm{M}$ a metal like $\mathrm{Nb}$ or $\mathrm{Ta}$ which has a $5+$ closed-shell configuration). The cation vacancy (V) centres are traps for hole polarons, and have corresponding descriptions. For a general survey of polarons see Stoneham (1989a) and Hayes and Stoneham (1985). The competition between different energy terms defines the regimes of behaviour for an electron coupled to a lattice distortion through scaling arguments. Whether or not a small polaron forms depends on the relative magnitudes of the lattice relaxation energy for the localized carrier $(S)$ and the transfer energy $(T)$. Looking beyond polaron behaviour to more general situations, a third energy, the on site coulomb repulsion (U) between two carriers, becomes important (Toyozawa 1981). Whether or not a small polaron has formed in practice is usually decided by transport measurements or spectroscopy, although other arguments are used. The transport approach is based on the increase of mobility of small polarons with temperature 
(whereas the carriers in metals or semiconductors have mobilities which fall as thermal scatter increases) and on the relation between the characteristic Arrhenius energy for motion and that from thermopower. The spectroscopic approach normally seeks a broad intrinsic absorption with a specific relation between energy and width. Oxides can be divided with reasonable certainty into small- and large-polaron forms by both the temperature dependence and absolute value of the carrier mobility (figure 1). Measurements of conductivity alone are far less informative, since changes in carrier numbers also often show Arrhenius behaviour.

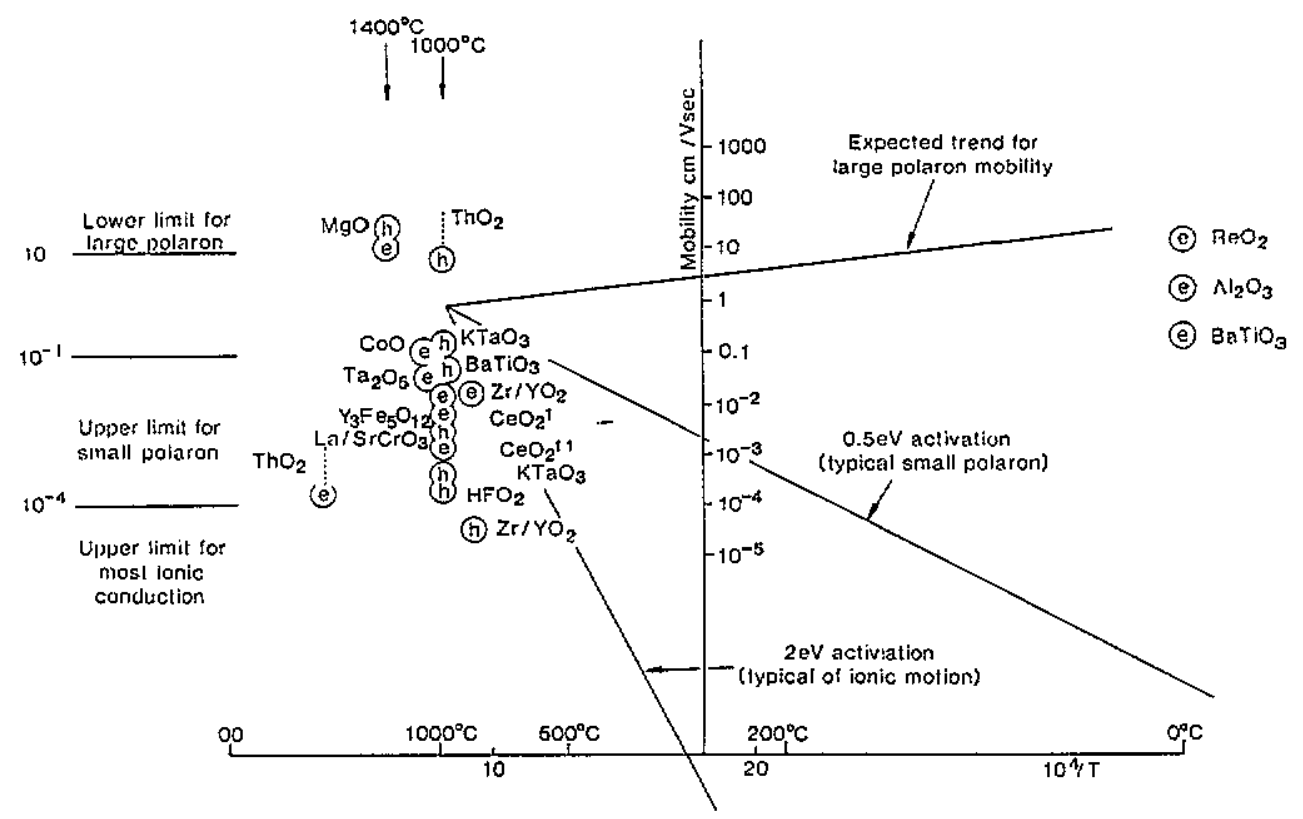

Figure 1. Observed electron (e) and hole (e) mabilities in oxides.

The information on non-superconducting ternary oxides is very incomplete, and often is based on ill-characterized samples. The general picture is that strontium titanate has heavy large-polaron states; it is not clear if the polaron is close to selftrapped or is defect-trapped. Small polarons are produced in lithium niobate. The following optical absorptions and proposed centres have been deduced for $\mathrm{LiNbO}_{3}$ (Arizmendi et al 1984).

$\begin{array}{llll} & \begin{array}{l}\text { Peak energy }(\mathrm{eV}) \\ \text { (Band width }(\mathrm{eV}))\end{array} & \\ \text { Species } & \text { Reduced crystals } & \text { Irradiated crystals } & \text { Species } \\ \text { Polaron } & 1.6(0.78) & 1.6(0.78) & \text { Polaron } \\ \text { Trapped hole } & 2.5(1.66) & 2.6(1.58) & \mathrm{F}^{+} \\ \text {Trapped hole } & 3.2(0.60) & 3.2(0.58) & \mathrm{F}^{0}\end{array}$

In barium titanate, holes are predicted (Lewis 1983) to bind strongly to Ti vacancies, and more weakly to $\mathrm{Ba}$ vacancies, whereas oxygen vacancies seem far less attractive to electrons. Boron-doped niobia, which lacks a metal-oxygen layer structure, appears to be a small polaron conductor which, despite high hole concentrations, 
shows no signs of superconductivity down to $4.2 \mathrm{~K}$ (Vaidya et al 1988). The electrical conductivity of $\mathrm{NbO}_{2}$ shows a remarkable increase with the addition of boron and a change from semiconductor-like behaviour to metal-like behaviour (see Lewis 1983, p 144). A survey (Stoneham 1989a) of polarons and bipolarons in oxides (discussed later) shows that small polarons appear to be present in many systems in which ions can change charge state (assuming that to be well-defined) without large energy demands. Hopping conductivity is also seen in precursor glasses of the BISCCO superconductors (Ghosh and Chakravorty 1990).

Small polarons in superconducting oxides are directly observable only in the normal state, though the possible role of small bipolarons in superconductivity cannot be ignored. In the temperature range from $650^{\circ} \mathrm{C}$ to $850^{\circ} \mathrm{C}, \mathrm{YBCO}$ (with 6.15 to 6.75 oxygens per unit) appears to be a small polaron conductor with a $0.1 \mathrm{eV}$ activation energy, similar to values reported in other oxides. The simplest fit of the data assumes copper in its $3+, 2+$ and $1+$ states. The equilibrium constant of disproportionation of the $\mathrm{Cu}^{2+}$ state into $\mathrm{Cu}^{+}$and $\mathrm{Cu}^{3+}$ appears to be independent of temperature and oxygen concentration (Su et al 1988).

3.2.2. Relationship to excitons. The most direct approaches to electronic excitation in semiconductors or insulators are from the temperature dependence of electrical conductivity, or from optical absorption or luminescence. We now comment on such experiments in oxides relevant to superconduction. With calculations based on the shell model, i.e. effectively a small polaron model, Lewis (Lewis 1983, p 128) has estimated the band gap in barium titanate, assuming that electron transfer occurs between the oxygen and titanium ions. This transfer corresponds to exciton formation or to electron-hole pair generation, depending on the extent to which lattice polarization is allowed and on the proximity of electron and hole. The value found is $3.05 \mathrm{eV}$, agreeing favourably with values from the observed temperature dependence of the conductivity. Thus the barium titanate will be a poorly conducting material at room temperature unless donor levels exist close to the conduction band. In fact, at low oxygen partial pressures, oxygen vacancies are the preponderant defect species, and shallow donor levels are present, leading to n-type conduction.

Luminescence is a very powerful tool, though prone to sensitivity to defects and impurities. For YBCO, synchrotron radiation studies (Stankevich et al 1988) in the energy range 4-30 eV show a broad luminescence of an exciton type at $2.8 \mathrm{eV}$ whose intensity shows structure at the temperature of the superconducting transition. This intensity decreases with a further increase in temperature. This may relate to data from quite different experiments, namely the thermally stimulated and $x$-ray induced luminescence of nominally undoped $\mathrm{YBa}_{2} \mathrm{Cu}_{3} \mathrm{O}_{7}$ (Roth et al 1988). In the low-temperature $\mathrm{X}$-ray luminescence spectrum, there is an intense band peaking at $2.8 \mathrm{eV}$. A comparison with existing data on $\mathrm{GdBa}_{2} \mathrm{Cu}_{3} \mathrm{O}_{7}$ and simple oxides suggests that any defects involved in the $\mathrm{x}$-ray induced luminescence are intrinsic in nature and possibly related to oxygen vacancies. A further $3.65 \mathrm{eV}$ thermally stimulated emission could be intrinsic or extrinsic.

Cathodoluminescence has been seen in YBCO at $3.355 \mathrm{eV}$, with half-width of only $3.5 \mathrm{meV}$ and lifetime less than $3 \times 10^{-9} \mathrm{~s}$ (Luschik et al 1987). The quantum efficiency was small, around $10^{-4}$. Above $60 \mathrm{~K}$ the luminescence is quenched with an activation energy of $24 \mathrm{MeV}$. This luminescence is interpreted as being due to recombination of electrons with a group of paired holes. The activation energy for quenching suggested to be the gap width for the superconducting state. A wide-band luminescence of $3 \mathrm{eV}$ 
was also observed, excited at $80 \mathrm{~K}$ by photons with energies above $5 \mathrm{eV}$.

The photo-induced optical absorption spectrum of $\mathrm{La}_{2} \mathrm{CuO}_{4}$ shows two peaks at $0.5 \mathrm{eV}$ and $1.4 \mathrm{eV}$, with a crossover to photo-induced bleaching at roughly $2 \mathrm{eV}$ (Ginder et al 1988). These data, together with the observed luminescence at about $2 \mathrm{eV}$, confirm the presence of an energy gap at or above this energy, as well as the existence of long-lived stable electronic defect states in this system.

Optical data for a series of $\mathrm{La}_{2-x} \mathrm{Sr}_{x} \mathrm{CuO}_{4-y}$ solid solutions from $x=0$ to $x=0.3$ reveal several structures, including a relatively intense, dopant-induced absorption band near $0.5 \mathrm{eV}$ whose oscillator strength varies systematically with both the Meissner effect and the critical temperature (Etemad et al 1988). The dopant-induced optical-transition correlation with superconductivity favours a mechanism of superconductivity in which there is an electronically driven pairing mediated by the $0.5 \mathrm{eV}$ excitation. The $0.5 \mathrm{eV}$ excitation itself could be a Jahn-Teller excitation. Further confirmation is needed.

Photoinduced absorption experiments on $\mathrm{YBCO}$ in the energy range 150$4000 \mathrm{~cm}^{-1}$ (Kim et al 1988) indicate photoinduced infra-red active vibrational modes, associated phonon bleachings and an electronic transition from a self-localized gap state. The results imply a local tetragonal to orthorhombic distortion around the charge carriers. This photoinduced structural distortion and the associated selflocalized gap state suggest that the photoexcitations are polarons or bipolarons. Since these photoinduced absorption features are not seen in the isostructural compound $\mathrm{La}_{2} \mathrm{NiO}_{4}$, the results suggest further that polaron or bipolaron formation may play an important role in the high temperature superconductivity.

A further link between optical features and superconduction is shown by observed onset of special photoconductivity coincident with the onset of superconductivity (Masumi et al 1987). The photoconductivity spectra of $\mathrm{Y}_{1} \mathrm{Cu}_{2} \mathrm{O}_{z}$ and $\mathrm{Y}_{3-x} \mathrm{Ba}_{x} \mathrm{Cu}_{3} \mathrm{O}_{9-y}$ appear to indicate polarons and excitons in the $\mathrm{Cu}_{2} \mathrm{O}$-like phase in these oxygen deficient systems. Again, this suggests that polaron and exciton phenomena must be important in the mechanism of superconductivity.

\subsection{Impurity centres}

One of the early successes of oxide superconductor synthesis was the progressive increase of critical temperature by doping, whether to control the carrier concentration, to provide an effective 'internal pressure' or to improve crystal quality. Much of the work was apparently intuitive (or even haphazard), though one should respect the intuition of experienced crystal chemists just as one does that of a master chef in his own recipes. Other work was specific in aim, seeking control of site occupancies for exploitation at some later stage. Not all changes are beneficial, of course; in BISCCO, for instance, all $3 \mathrm{~d}$ dopants ( $\mathrm{Fe}, \mathrm{Co}, \mathrm{Ni}$ and even $\mathrm{Zn}$ ) seem to decrease the critical temperature (Maeda et al 1990).

In barium titanate, monovalent ions can be incorporated into the lattice at barium or titanium sites, charge-compensated by hole formation or the production of oxygen vacancies. The various possibilities can be assessed very effectively by theory, exploiting the ionic models noted earlier in discussing the 'reference crystal' concept for superconducting oxides. Calculations (Lewis 1983) suggest that in fact the alkali ions substitute at barium sites, with oxygen vacancy compensation. Isovalent substitutional ions ( $2+$ at barium sites and $4+$ at titanium sites) need no compensating defect. As with monovalent dopants, there is a strong correlation between the heat of solution and the ionic radius. Larger ions, whose radii correspond more closely to that 
of $\mathrm{Ba}^{2+}$, show a marked preference for substitution at these sites, whereas smaller ions (e.g. $\mathrm{Mg}^{2+}$ ) will substitute at least partially at titanium sites, even though additional charge compensating defects are needed. Trivalent dopants can substitute at barium sites, leading to an excess positive charge that is compensated by conduction electrons or metal vacancies. The trivalent ions can substitute at titanium sites, with oxygen vacancy compensation or electron-hole compensation.

A third possibility analysed theoretically is self-compensation, whereby a dopant simultaneously substitutes at both sites. Whilst there is a strong correlation between ionic radius and the tendency towards self-compensation for a particular dopant, a knowledge of the ionic radii alone is wholly inadequate to predict this substitution mechanism for any particular dopant. There are complications from both a dependence on coordination and possibly from electronic effects, and thus there are more delicate balances that need to be considered than merely ionic radii. The ionic models based on the shell model do, however, give a convenient and sufficiently accurate approach to assess self-compensation (Lewis 1983).

3.3.1. Intentional substitutions. In a systematic study of the effects of doping with transition and non-transition metal ions at copper sites (Kistenmacher 1988) the following conclusions emerge.

(i) Closed-shell dopants $(\mathrm{Ga} / \mathrm{Al}, \mathrm{Zn})$ show a limiting behaviour for effects on spontaneous strain and on changes in critical temperature.

(ii) Discrimination between occupancy of the $\mathrm{Cu}(1)$ and $\mathrm{Cu}(2)$ sites can be made by looking at the changes in spontaneous strain and critical temperature.

(iii) Selective doping applies to open-shell ions (trivalent $\mathrm{Fe}$ and $\mathrm{Co}$ at the $\mathrm{Cu}(1)$ site, and divalent $\mathrm{Ni}$ at the $\mathrm{Cu}(2)$ site), as well as to closed-shell ions (trivalent $\mathrm{Ga}$ and $\mathrm{Al}$ at the $\mathrm{Cu}(1)$ site and divalent $\mathrm{Zn}$ at the $\mathrm{Cu}(2)$ site). It appears that charge selectivity (presumably at higher temperatures, during the processing of the oxide) could be the direct antecedent of site selectivity in these chemically complex materials.

Exactly these results emerge for YBCO from ionic crystal calculations based on the shell model and empirical two-body interatomic potentials (Baetzold 1988). In addition, this theory predicts that both oxygen vacancy formation and hole trapping as a localized polaron are most favoured at the chain oxygen ion site $\mathrm{O}(1)$. Hole trapping on copper ions is predicted to be most favoured for ions in the copper-oxygen plane. Though divalent impurity ions (such as nickel) are predicted to dissolve in the crystal preferentially at the plane site $\mathrm{Cu}(2)$, trivalent impurities (such as aluminium) are predicted to dissolve in the crystal at the chain site $\mathrm{Cu}(1)$, along with the incorporation of oxygen ions. Anions such as $\mathrm{S}^{2-}, \mathrm{F}^{-}$and $\mathrm{Cl}^{-}$are predicted to substitute preferentially for those oxygens at sites near the copper chain positions.

It proves possible to substitute $\mathrm{Ca}$ at either the $\mathrm{Y}$ site or the $\mathrm{Ba}$ site in $\mathrm{YBCO}$, depending on the precise method of preparation (Chandrachood et al 1990). Substitution at the $\mathrm{Ba}$ site leads to a tetragonal structure in which superconductivity is suppressed (Baldha et al 1989); substitution at the $Y$ site destroys neither superconductivity not the orthorhombic structure.

Unexpected impurities can enter during the preparation stages. Laser spectroscopy of a $\mathrm{Eu}^{3+}$ probe ion identifies two impurity phases in polycrystalline samples of $\mathrm{La}_{1.85} \mathrm{Sr}_{0.15} \mathrm{CuO}_{4}$ (Tissue and Wright 1988). These phases are residual hexagonal $\mathrm{La}_{2} \mathrm{O}_{3}$ starting material, and a La-silicate phase which forms during sintering from contamination with boat or furnace material. The laser spectroscopy of the $\mathrm{Eu}^{3+}$ 
probe provides a useful monitor of the solid state reaction rate of the starting materials, as well as showing up heterogeneities in the distribution of the impurity phases in the final samples.

9.3.2. Comparison with normal ionics. In lithium niobate, all disorder is on the cation sublattice, with $\mathrm{Nb}$ or $\mathrm{Li}$ antisite defects. Bound bipolarons are especially associated with the disorder, for example the bipolaron including one $\mathrm{Nb}$ on a $\mathrm{Li}$ site and one on an $\mathrm{Nb}$ site. Lewis's work on $\mathrm{BaTiO}_{3}$ (Lewis 1983) shows that here the large difference in ionic radii tends to give strong site dependence for impurities. For $\mathrm{SrTiO}_{3}$ there is a less clear-cut distinction between the ion sizes, and so there is a tendency for trivalent ions to substitute almost equally on $\mathrm{Sr}$ and $\mathrm{Ti}$ sites, so giving self-compensation and causing it to be an insulator overall.

Similar factors may be involved in excited states. Experimental results for strontium titanate under pulsed electron irradiation show some interesting differences from behaviour in binary oxides (Tanimura et al 1984). Volume changes are induced in quartz, strontium titanate and $\mathrm{CaO}$ and $\mathrm{MgO}$ by the pulsed electron beam, and those seen for strontium titanate are similar to those for quartz. Previous experiments on quartz showed that a photolytic reaction (roughly equivalent to a creation of defects by electronic excitation) gave the observed transient volume change. Tanimura et al suggest that a photolytic reaction occurs in strontium titanate too, although the effect may possibly be extrinsic. The volume change in $\mathrm{MgO}$ or $\mathrm{CaO}$ is ascribed to the trapping of electrons or holes by existing defects.

Doping by ion-implantation and subsequent annealing is an alternative to the usual equilibrium routes. One example which shows site preferences (Farlow et al 1984 ) is for metal ions ( $\mathrm{Fe}, \mathrm{Mn}, \mathrm{Ni}, \mathrm{Cr}$, and $\mathrm{Ga}$ ) implanted at random orientations into single crystals of alumina. These crystals were then annealed in flowing oxygen at temperatures between 600 and $1500^{\circ} \mathrm{C}$. Analysis showed that the normally trivalent ions ( $\mathrm{Fe}, \mathrm{Ga}, \mathrm{Cr}$ ) had a strong tendency to be incorporated in substitutional sites, whereas the normally divalent ions $(\mathrm{Cu}, \mathrm{Mn}$ ) showed no such tendency, even at high temperatures. Most of the impurities tend to move towards the surface, with only the trivalent $\mathrm{Fe}, \mathrm{Ga}$, and $\mathrm{Cr}$ showing any tendency towards bulk diffusion. In all cases the aluminium sublattice recovers at lower temperatures than the oxygen sublattice.

For strontium titanate, substitutions on the titanium site have been identified for many different charge states of iron-group ions: for $\mathrm{Cr}$, for instance, the $5+$ and $3+$ states (the $4+$ is probably present but not seen), and for the higher- $Z$ elements like $\mathrm{Ni}$, the $4+, 3+$ and $2+$ states; for Fe four states $(5+$ through $2+)$ are observed. This wide range of stability is not due to covalency, but to the substantial polarizability of the lattice (Hayes and Stoneham 1985). However, this electron-lattice coupling is too weak to lead to negative $U$ behaviour (charge disproportionation). This has been examined in detail theoretically both for normal ionics (Stoneham and Sangster 1983) and for the La superconducting oxides (Catlow et al 1988, Zhang et al 1990) in work which considered possible hole coupling mechanisms. Negative $U$ processes (charge disproportionation) for both the $\mathrm{Cu}(3 \mathrm{~d})$ and $\mathrm{O}(2 \mathrm{p})$ bands were energetically unfavourable. Though this work concentrated on the La compound, the arguments have more general implications for oxide superconductors.

Doped $\mathrm{La}_{2} \mathrm{CuO}_{4}$ contains divalent ions substituted for the $\mathrm{La}^{3+}$ host cations, with charge compensation by hole formation. Catlow et al considered how these holes might pair. Impurities $\mathrm{M}^{n+}$ sitting in host cation sites of charge $+m(m>n)$ have to be compensated to ensure overall electrical neutrality. This is often through an oxygen 
vacancy, and indeed all perovskites show evidence of low-energy oxygen vacancy diffusion mechanisms. The vacancy may be bound to the impurity forming a complex, such as the $M n+n-V^{0}$ centre. For perovskite crystals a large number of complexes have been identified by spin resonance. These are listed below (Arizmendi et al 1984):

$\begin{array}{lllll}\text { Vac/imp complex } & \mathrm{SrTiO}_{3} & \mathrm{BaTiO}_{3} & \mathrm{PbTiO}_{3} & \mathrm{LiNbO}_{3} \\ \mathrm{Vac}+\mathrm{M}^{4+} & \mathrm{Fe} & & & \\ \mathrm{Vac}+\mathrm{M}^{3+} & \mathrm{Mn} \mathrm{Fe} \mathrm{Ni} & \mathrm{Fe} & \mathrm{Fe} & \mathrm{Ti}, \mathrm{Fe} \\ \mathrm{Vac}+\mathrm{M}^{2+} & \mathrm{Mn}, \mathrm{Co} & & & \mathrm{Mn}, \mathrm{Co} \\ \mathrm{M}^{3+}+\mathrm{e}+\mathrm{vac} & \mathrm{Ni} & & & \\ \mathrm{M}^{3+}+2 \mathrm{e}+\mathrm{vac} & \mathrm{Ni} & \mathrm{La} & & \\ \mathrm{M}^{2+}+\mathrm{e}+\mathrm{vac} & & \mathrm{Ba} & & \end{array}$

3.3.9. Modelling. We now turn to further modelling studies of superconducting and related oxides. These cover structures, lattice vibrations, and a range of other phenomena parallelling earlier work on non-superconducting oxides.

Lewis has done considerable work on computer modelling of mixed oxides, particularly barium titanate, investigating the substitution of monovalent, isovalent and trivalent ions at both $\mathrm{Ba}$ and $\mathrm{Ti}$ sites. He found that monovalent ions substitute at the $\mathrm{Ba}$ sites with oxygen vacancy compensation. Substitution with hole formation is energetically less favourable. Sodium has the optimal ionic radius. The divalent ions with a larger radius, corresponding more closely to that of $\mathrm{Ba}$, show a marked preference for substitution at that site; smaller divalent ions will substitute at least partially at $\mathrm{Ti}$ sites, even though additional charge compensating defects are necessary. For the smaller trivalent ions (of radius less than that of $\mathrm{Sc}$ ) substitution at $\mathrm{T} i$ sites is energetically favourable. Intermediate size trivalent ions favour self-compensation and large trivalent ions favour substitution at $\mathrm{Ba}$ sites. At high dopant concentrations, self-compensation becomes more effective. Tetravalent dopants favour substitution at $\mathrm{Ti}$ sites, with $\mathrm{Mn}$ having the optimal radius. It is clear that there is a strong ion size effect in the choice of substitution site, but care must be exercised, because the situation is more complex, and other factors (notably polarization energies, including those of charge-compensating species) are involved.

Islam et al (1988) have reported results of simulation studies of the pure and doped lanthanum compound which give insight into problems concerning cation valence states, defect structure, redox processes, the nature of electron-lattice interactions and electron-electron coupling mechanisms. The interatomic potentials used were taken from the relevant binary oxides e.g. $\mathrm{La}_{2} \mathrm{O}_{3}, \mathrm{BaO}, \mathrm{SrO}$ and $\mathrm{CuO}$. They found that a satisfactory model needed to go beyond the usual pair-potential approximation. The resultant equilibrium structure for $\mathrm{La}_{2} \mathrm{CuO}_{4}$ is close to that observed experimentally. Calculated phonon dispersion curves show a softening of one of the branches along the $\mathrm{b}$ direction of the unit cell. The direction of this soft phonon mode is along the long $\mathrm{O}-\mathrm{Cu}-\mathrm{O}$ axis. It involves movement of $\mathrm{La}$ and $\mathrm{O}$ ions only. Calculated electronic and displacement polarization energies are substantial (about $3 \mathrm{eV}$ ) indicating the strength of electron-lattice interaction.

A survey of defect energies finds that doping of $\mathrm{La}_{2} \mathrm{CuO}_{4}$ with divalent cations leads to oxygen vacancy compensation, but that oxidation of the resulting compounds is exothermic with the creation of trivalent copper ions. An examination of vacancy 
energies shows it is energetically more favourable to form oxygen vacancies in equatorial, rather than axial, positions. This accords with experiments on YBCO. It is found to be energetically favourable to form trivalent $\mathrm{Cu}$ rather than monovalent $\mathrm{O}$, but this does depend critically on the assignment of the second oxygen electron affinity, which (even for conventional oxides) is always in doubt. The copper disproportionation reaction from $\mathrm{Cu}^{2+}$ into $\mathrm{Cu}^{3+}$ and $\mathrm{Cu}^{+}$forms is found to be energetically unfavourable. The calculated solution energies of $\mathrm{SrO}$ and $\mathrm{BaO}$ in the $\mathrm{La}$ compound are accord with the observation that divalent oxides dissolve readily in $\mathrm{La}_{2} \mathrm{CuO}_{4}$. In other unpublished work, vacancy binding to $\mathrm{Sr}$ has been reported.

In YBCO, predictions (Islam and Baetzold 1989) suggest that the in-plane cupric site is favoured by divalent $\mathrm{Ni}, \mathrm{Zn}$ and $\mathrm{Cd}$, whereas the divalent alkaline earths $\mathrm{Ca}$ and $\mathrm{Sr}$ prefer the $\mathrm{Ba}$ site. The situation is less clear-cut for trivalent ions; lanthanides are predicted to prefer the $\mathrm{Ba}$ site, but $\mathrm{Al}$ and $\mathrm{Fe}$ chose a $\mathrm{Cu}$ site, which one depending on what is assumed about the nature of the free carrier (here if an $\mathrm{Al}$ atom and a $\mathrm{Cu}$ atom are exchanged, there will be an extra electron if the final states are $\mathrm{Cu}^{2+}$ and $\mathrm{Al}^{3 \div}$ ).

Bipolarons have attracted attention as bosons which might condense into a superconducting state. Whether or not this is the mechanism of superconductivity, the idea is both consistent with evidence for bipolaron formation in reduced $\mathrm{WO}_{3}$, reduced $\mathrm{LiNbO}_{3}$, and oxides involving $\mathrm{Nb}$, Ti and $\mathrm{W}$ (for references and comment see Stoneham 1989a) and with some, at least, of the systematics of oxide superconductors. Islam et al investigated the interaction between $\mathrm{Cu}^{3+}$ ions to see if they may couple to form bipolarons; similar work has since been reported (Catlow et al 1988, Baetzold 1988). None of the hole pairs are found to be bound, but the polarization energy strongly favours coupling of the pairs, and screening effects could reduce the coulomb repulsion energies to result in stable bipolarons (in fact, these calculations also omit the Jahn-Teller contribution to pairing, estimated e.g. by Stoneham (Stoneham 1987). In their paper, Catlow et al (1988) consider further hole-pairing mechanisms in $\mathrm{La}_{2} \mathrm{CuO}_{4}$. They conclude that a peroxy-type of bipolaron species is stable by about $0.2 \mathrm{eV}$ (for the configuration they call equatorial-equatorial). Bipolarons are close to stable and with a little Jahn-Teller effect could well become so.

In a very wide-ranging series of calculations, Allan and Mackrodt (1988a,b, 1989) have made theoretical calculations of the perfect lattice and defect properties of $\mathrm{M}_{2} \mathrm{CuO}_{4}$ (M $\equiv \mathrm{La}, \mathrm{Pr}, \mathrm{Nd}, \mathrm{Al}$ ). The principal aim was to explain why $\mathrm{M} \equiv \mathrm{La}$ leads to superconducting materials, whereas the remainder do not. Their calculations, based on interatomic potentials largely obtained from electron-gas calculations, were able to correctly predict the observed crystal structures for all of these systems. This is itself an achievement, since the several alternative structures are close in energy. The predicted phonon density of states based on these relatively simple electron-gas potentials proves to be a useful investigative 'fingerprint' in the assessment of potential superconducting oxides.

Many substitutions were studied in this work, showing again that ion size is important. For example, since $\mathrm{Cu}$ has a small ionic radius and $\mathrm{La}$ a large one, of all the alkaline earths only $\mathrm{Mg}$ might substitute at a $\mathrm{Cu}$ site. Holes are predicted to tend to settle on $\mathrm{Mn}, \mathrm{Fe}, \mathrm{Ni}$ and $\mathrm{Co}$ rather than on $\mathrm{O}$. Dopants from the Fe group should substitute at either the $\mathrm{Cu}$ sites or the alkaline earth sites.

3.3.4. Substitution on the anion site. There have been several reports of anion dopants affecting superconducting behaviour. Thus Felner et al (1987) have shown that the 
substitution of one oxygen in YBCO with sulphur does not change the critical temperature, but the sulphur-doped sample has an even sharper transition, and its Meissner effect is almost complete. Similar results were obtained with substitution of oxygen with small quantities of fluorine (Hakwraku et al 1988). Palhan et al (1988) sought to discover if the sulphur was in the solid matrix as a substitutuent or whether it segregated to defects and acted as a catalyst to strengthen the superconducting coupling among the grains. Samples with sulphur were found to be unstable, decomposing after several weeks to barium sulphate and other phases. Electron microscopy identified a sulphur-containing phase of approximate composition $\mathrm{YBa}_{2} \mathrm{Cu}_{3} \mathrm{O}_{6} \mathrm{~S}$ on fresh samples. This phase was not twinned, suggesting that the absence of the twin boundaries is responsible for the larger Meissner effect. This phase was not observed in old samples, so it is probably not thermodynamically stable. Palhan et al cite Mossbauer measurements on the related Eu-based systems. The larger quadrupole splitting seen in the sulphur-doped case is attributed to substitutional sulphur. In fluorine-doped YBCO (LaGraff et al 1989) prepared by a $\mathrm{NF}_{3}$ gas anneal, $\mathrm{x}$-ray and neutron diffraction studies showed that the fluorine is structurally incorporated into the $\mathrm{CuO}$ chains, in the normally vacant $O(5)$ sites along the $a$ axis. This results in a decrease in the orthorhombic strain. As the fluorine content is altered, the critical temperature remains the same, but diamagnetic expulsion improves significantly.

\subsection{Unintentional and intentional contaminants}

Unintended contaminants can enter in many ways. One is through substrate/contact interactions, which will be discussed later. Another source of contaminants is through atmospheric interactions, i.e. interaction with water and carbon dioxide, again discussed later. These phenomena are widespread in oxdes (Freund et al 1984). However, some contaminants have later shown to be beneficial, possibly because they limit the extent of atmospheric degradation. In particular, the effects of silver have been investigated by many workers, and we shall return to discuss this point later. In one study (Peterson et al 1988) x-ray photoelectron spectroscopy, scanning Auger and optical microscopy of polycrystalline superconducting pellets of $\mathrm{YBCO} / \mathrm{Ag}$ showed the silver-laced samples to have a lower porosity and a drastically reduced hydrocarbon contamination. The results indicate that there is no detectable substitution of $\mathrm{Ag}$ into the $\mathrm{YBCO}$, but metallic silver collects in voids and possibly along grain boundaries. Intergranular silver may well mitigate adverse grain boundary effects in polycrystalline YBCO.

\section{Surfaces and interfaces}

The main structural, electrical and defect properties of ceramic interfaces have become much clearer with the development of reliable theoretical modelling methods (Tasker and Stoneham 1984, Hayes and Stoneham 1985). Such calculations have led to the understanding of many phenomena-for instance the nature of the tilt grain boundary (Tasker and Dufour 1988) or the main features of bonding between an oxide and a metal (Stoneham and Tasker 1989)-and have also been the subject of very stringent quantitative tests, including the effects of surfaces on the phonon density of states (Hoare et al 1983). Among the several features of these ionic interface studies relevant to superconductors one might include the following. 
(i) Diffusion in grain boundaries: Is it enhanced, as in normal ceramics? Can this enhancement be diminished by doping to exploit the so-called 'reactive element effects?'

(ii) Many ceramics show ready amorphization at grain boundaries. Is this typical of superconductors? In fact, the issue of which oxides go amorphous, whether there is one or more amorphous phase, and which properties are altered on amorphization is still hard to answer. Clearly there is much information on formation of simple glasses. But are there any superconducting glasses? In many theoretical models, the answer would seem to be yes; none seem to be known, although superconductors derived from glass ceramics are reported.

(iii) By what mechanism do planar (or other) defects pin flux lines? If pinning is strong (as seems to be the case) does this rule out defect-mediated carrier pairing? Is intrinsic pinning possible, e.g. like polaron self-trapping, but arising perhaps from the magnetostriction of the normal regions? The normal and superconducting regions of a flux line must have some means of interaction with the structural or magnetic changes due to lattice defects. The direct electronic interaction seems too small, so that a more subtle-but bigger-interaction is needed.

(iv) What features of grain boundaries give limits on intergrain supercurrents and determine their relation to the grain boundary angle? Is it the fact that dislocation cores are normal (non-superconducting) and that the fraction of normal phase along the boundary is critical? If so, since it is possible to model the grain boundaries, can one use the derived geometries in some simple way to predict the intergrain supercurrent?

(v) The adhesion of metal to superconductor is important for current bypasses and for contacts, both electrical and thermal. Can the adhesion be enhanced by the same methods that work for normal ceramics, as for radiation-enhanced adhesion?

(vi) There can be important planar structure within grains. Thus it has been argued (Khachaturyan and Morris 1990) that, in YBCO, the oxygen vacancies are assimilated rather as in $\mathrm{TiO}_{2-x}$ or $\mathrm{MoO}_{3-x}$, where the Magneli phases are found. In YBCO the series of transient ordered structures represents various ways of ordering atoms, vacancies in the basal plane. As $\mathrm{O}$ is removed by bulk diffusion, reorganization occurs by local diffusion and the orthorhombic phase changes systematically until it spontaneously disorders into the tetragonal phase. Such vacancy assimilation will affect both the phase boundary between the orthorhombic and tetragonal phases and what diffusion process is actually measured in some experiments. Distinctions between local and long-range diffusion are noted elsewhere.

\subsection{Free surfaces}

The situation for non-superconducting oxide surfaces is now fairly well defined (Dufour and Nowotny 1989). What is known in those cases suggests that, for superconducting oxides the key questions here are: What atomic species provide the termination? Is there any significant reconstruction? In many cases (noted below) there is evidence of altered surface layers, either from segregation or from some artefact of manufacture (e.g. exposure to a reactive atmosphere). In the absence of such phases and if oxygen excess or loss is avoided, the picture seems to be this:

(i) STM data show that the bulk orthorhombic structure of YBCO extends right up to the surface. In one case, at least, there is no real sign of semiconducting behaviour at the surface (van de Leempt et al 1988). 
(ii) For at least some types of YBCO grain, full image reconstruction suggests that in $\mathrm{YBCO}-\mathrm{BaO}-\mathrm{BaO}$ - surface layers predominate and that these layers and adjacent -CuO-CuO- layers suffer oxygen depletion (Bursill and Fan 1988).

(iii) Not all YBCO grains are of the same type. Auger scanning microscopy experiments (Cota et al 1988) show that two phases are present. One is the standard compositional phase (this shows a platelet shape) and the second phase (which has a granular appearance) is $\mathrm{Ba}$ enriched.

\subsection{Other interfaces}

4.2.1. Surface phases. Both the La compounds and the YBCO compounds are unstable against reaction with atmospheric water and carbon dioxide (noted previously). These reactions are common too in non-superconducting oxides, and indeed reactions at grain boundaries are exploited in solid-state gas sensors. Such reactions can be chemical, or they may require only electron transfer between adsorbed species and substrate (Moseley and Tofield 1987); in other cases, impurity segregation to grain boundaries may be critical. Clearly, any technique which causes surface modification can influence the extent of atmospheric attack, varying from controlled segregation to ion implantation, where it has been noted both for conventional glasses (Chengyu $e t$ al 1987) and YBCO (Chaudhari et al 1989) that nitrogen ion implantation reduces aqueous degradation.

Ultraviolet photoemission experiments (Flavell and Egdell 1988) show the formation of barium carbonate and $\mathrm{CuO}$, other products being a different $\mathrm{Y} / \mathrm{Ba} / \mathrm{Co}$ oxide and oxygen gas. The loss of superconductivity in YBCO on exposure to moisture and its variation with both the oxygen content of the superconducting phase and the phase mixture of the specimen shows a phase transition occurred to tetragonal structure. This behaviour (Harris and Nyang 1988) was explained by the rapid diffusion of water molecules along the channels used for oxygen intercalation, leading to the eventual reaction of water with barium ions. ESR measurements (Cywinski et al 1988) showed no room temperature signal in the region $g=2$ though, on exposure to the atmosphere, a signal appeared, the intensity and lineshape of which evolved with time. This signal was attributed to atmospheric degradation of the superconducting ceramic splinters. Many other authors find analogous results. If the $\mathrm{Y}$ of $\mathrm{YBCO}$ is substituted by $\mathrm{Zr}$ or $\mathrm{Hf}$ in a regime where all samples remain superconducting above liquid nitrogen temperatures (Regnier et al 1988), the resistance to humidity can be made to increase significantly. Photoemission results for YBCO (Qiu et al 1988) show that water modifies valence band states, reduces the number of inequivalent $\mathrm{Ba}$ sites and induces two $O$ 1s core-level components at 534 and $531 \mathrm{eV}$. The $534 \mathrm{eV}$ component is coincident with that of cryosorbed ice and the enhanced $531 \mathrm{eV}$ component is due to a new water-induced bonding environment in the superconductor. This is most probably a hydroxyl species. The changes in the Ba core-level appearance suggest that water may be preferentially modifying $\mathrm{YBCO}$ in a way that affects the $\mathrm{Ba}$ atomic environment. In the light of their results, Qiu et al conclude that previous reports suggesting oxygen dimerization (cf our section on oxygen defects) below the superconducting transition, as well as some other reported changes in the spectra, are improbable.

Since superconducting properties degrade with time on exposure to air, it is therefore very important to identify and separate the segregates which appear under various preparation conditions. For this reason Muraleedharan et al (Muraleedharan et al 1988 ) performed infra red studies on $1: 2: 3$ oxides. They detected the precipitation of 
barium carbonate during the slow cooling of the samples in the conventional ceramic technique, and concluded that this may explain the reported presence of carboxylic groups in $\mathrm{YBCO}$ in photoelectron and photoemission spectroscopy. Also discussed was the possibility of a slow degradation of superconducting properties by barium carbonate precipitation at room temperature by reaction of $\mathrm{YBCO}$ with $\mathrm{CO}_{2}$. For the $\mathrm{Tl}$ compound, resonant photoemission (Stockbauer et al 1989) shows that water reacts to form hydroxide (as in many conventional oxides) and carbon dioxide to form carbonate, both with a sticking probability of around 0.2 ; molecular oxygen, however, does not react, and $\mathrm{CO}$ reacts only weakly. There is a dependence on oxygen level of YBCO of the manner of interaction with water and hydrogen (Ruckman et al 1989). The stoichiometric oxide with 7 oxygens per formula unit shows only slow degradation, with water molecules (or some hydrogeneous species) penetrating the bulk before reaction; with less oxygen (6.2-7 per formula unit) the reaction is more rapid: a ten minute exposure to water completely decomposes YBCO containing as few as 0.2 oxygen vacancies per unit cell.

It is interesting to compare results for $\mathrm{MgO}$ (Freund et al 1984) which show that single crystals of $\mathrm{MgO}$ take up traces of water and carbon monoxide or dioxide into solid solution during crystal growth. The water was found to yield an equimolal concentration of extrinsic cation vacancies and hydroxyl ions. Fully hydroxyl- compensated cation vacancies were found to convert to a large extent into molecular hydrogen and peroxy ions. The hydrogen may be lost, leaving peroxy ions as excess oxygen. The thermal decay of the peroxy ions above $530^{\circ} \mathrm{C}$ appears to yield free positive holes, both vacancy bound $\mathrm{O}^{-}$states and highly mobile, unbound holes on the oxygen sublattice which diffuse to the surface. They then release oxygen, set up a surface charge and also cause auto-oxidation of bulk transition-metal impurities. The carbon does not occur as carbonate ions, but as $\mathrm{CO}_{2}^{2-}$ and $\mathrm{CO}^{-}$, with the carbon being very reduced, essentially zero-valent. The solute carbon seems to have an unusually low activation energy for diffusion and, even at low temperatures, has a strong tendency for subsurface segregation.

Surface-sensitive spectroscopies also show modified layers involving the metal sublattice. Thus there is evidence (Healy et al 1988) for the migration of barium to the free surface during heat treatment, attributed to thermal diffusion along the grain boundaries (Cerezo 1989, Schrott et al 1989). High-resolution electron spectroscopy measurements (Zandbergen et al 1988) on YBCO before and after heating at various temperatures and in various atmospheres show that in air, oxygen, and vacuum the surface decomposes rapidly for temperatures above about $423 \mathrm{~K}$. Below this temperature the rate of decomposition is much lower. The decomposition reaction proceeds by the insertion of extra $\mathrm{CuO}$ planes into the original structure, resulting in $(\mathrm{CuO})_{2}$ double layers between $\mathrm{BaO}$ planes. This induces large lattice strains at the exposed surfaces, leading to fragmentation and spalling. Again, such behaviour is common in non-superconducting oxides, where fragmentation can be efficient, with particle sizes close to the comminution limit (i.e. almost all the energy from oxidation is used to generate new surface).

Turning to the Bi-based superconductors, Kirk et al (1988b) have studied the crystal structure of $\mathrm{Bi}_{2} \mathrm{Sr}_{2} \mathrm{CaCu}_{2} \mathrm{O}_{8+x}$ which has an incommensurate superstructure along the $b$ axis of the material. The superstructure is believed to affect the superconductivity of the material, since there is a sizeable buckling of all planes including the $\mathrm{CuO}$ planes. Their atomic resolution images of the $\mathrm{BiO}$ plane suggests that a cause for the observed incommensurate superstructure is a row of missing $\mathrm{Bi}$ atoms every 
nine or ten atomic sites in both $\langle 110\rangle$ directions. A model is proposed that includes missing rows of atoms, as well as displacements of the atomic positions along both the $a$ - and $c$-axis directions. In an earlier paper (Kirk et al 1988a) the surface $(a, b$ plane) of the same oxide was seen to become insulating at low temperatures, exhibiting a large BCS-like energy gap. Related work concerns the effects of adsorbed Cu, a situation which is relevant to the formation of contacts. Hill et al (Hill et al 1988) have used XPS to examine the electronic structure and surface interactions for vapour deposited copper on single-crystal and polycrystalline $\mathrm{Bi}_{2} \mathrm{Ca}_{1+x} \mathrm{Sr}_{2-x} \mathrm{Cu}_{2} \mathrm{O}_{8+y}$. The $\mathrm{Cu}$ adatoms do not disrupt this oxide as extensively as $\mathrm{YBCO}$, but the deposition of $\mathrm{Cu}$ induces changes in the $\mathrm{Bi}$ environment in the superconductor. Surface segregation of $\mathrm{Bi}$ metal is observed at high coverages, but with minimal out-diffusion of oxygen.

Effect of noble metals. Many rare earths have been substituted for $\mathrm{Y}$ in $\mathrm{YBCO}$ to try to develop materials with superior superconducting properties. However, another class of additives, $\mathrm{Ag}$ and $\mathrm{Au}$, appears not to react at all with $\mathrm{YBCO}$. These metals have therefore been suggested as passivating layers for YBCO surfaces, to prevent the detrimental interactions with atmospheric carbon dioxide and water. Similar beneficial effects are found when $\mathrm{YBCO}$ is formed from layers of yttria, $\mathrm{Cu}$ and $\mathrm{BaO}$ deposited on a substrate with silver underneath or on top (Chang et al 1989).

The same metals, $\mathrm{Ag}$ and $\mathrm{Au}$, appear to help with other problems, namely mechanical strength (Singh $\epsilon$ t al $1989 \mathrm{a}, \mathrm{b}$ ) and the low critical currents in many bulk materials and even thin films. The effect on the strength may be a consequence of relief of residual stresses due to expansion anisotropy and an increase in resistance to crack propagation by crack pinning. The improved critical currents may be due to effects on the intergranular weak links at grain boundaries, where the noble metal may reduce the effects of interactions with atmospheric contaminants via surface-connected porosity. As noted earlier, intergranular silver does indeed appear to mitigate adverse grain boundary effects in YBCO (Peterson et al 1988). Improvements in thick films (Miller et al 1989) are attributed partly to enhanced grain growth, and partly to improved coupling from proximity effect electron pairing in the silver regions. Other effects are associated with $\mathrm{Ag}_{1}$ for example a reduction in workfunction of $0.3 \mathrm{eV}$ by submonolayer coverage (Schrott et al 1989).

4.2.2. Grain boundaries. We summarise first the geometric features of grain boundaries observed in $\mathrm{YBCO}$.

(i) High aspect-ratio grains have as long, flat, sides the basal planes and as the shorter sides mainly (010), (100) or (110) planes.

(ii) The grain boundary has a profound effect on the inter granular current. This can be rationalised approximately by saying that, for tilt boundaries, the dislocations are normal material, so that the superconducting part is reduced as the tilt angle rises.

(iii) In conventional oxides, the intergrain angles are not random, but are determined by interfacial energies and (often primarily) by contamination (see Hayes and Stoneham (1985), p 178, where the calculated energies for tilt boundaries based on a (100) plane of NiO are shown as a function of tilt angle (Tasker and Duffy 1983)). There is therefore potential for optimizing microstructure. The maximum energy observed in this plot is due to a balancing of two factors; the dislocation density along the boundary decreases with decreasing angle and the energy per dislocation increases with decreasing angle (Tasker 1983). From studies of misorientations adopted by grains which are free to rotate relative to one another, it appears that low-energy 
structures occur for cubic materials at those misorientations for which a coincidence site lattice (CSL) occurs. The volume of the CSL unit cell is larger than that of the crystal unit cell by a factor sigma; low sigma values are favoured (Smith et al 1988). The most favoured relative angles for cubic crystals, with rotations about the [100] axis, in order of decreasing popularity, are: $28.07^{\circ}, 43.69^{\circ}, 36.87^{\circ}, 12.68^{\circ}, \ldots$, (for the cubic case, with fourfold symmetry, the angles are defined to lie between $0^{\circ}$ and $45^{\circ}$ ).

(iv) One non-destructive way to characterize the microstructure of a ceramic superconductor exploits AC inductive measurements ( $\mathrm{Ni}$ et al 1988) to provide a distribution of grain sizes in YBCO. This distribution could be approximated by a function of the form

$$
P(g)=[q-1] g(q-1) \exp [-A g(q)]
$$

where the grain dimensions $g$ are expressed in terms of the mode of the grain size $G$ and the overall distribution is normalised;

$$
A=(q-1) / q \text {. }
$$

For these data $G=8.6 \mu \mathrm{m}, q=3.0$; an alternative fit is $G=4.2 \mu \mathrm{m}$ and $q=2.2$. Whichever is used, the major size is around $4 \mu \mathrm{m}$. Wen et al (1988) also give grain size distributions and other details of microstructure.

The character of the grain boundaries affects both the normal and the superconducting currents, as can be seen from a variety of theoretical studies (Choy and Stoneham 1990) and from the experimental studies to which we now turn.

Altered phases at grain boundaries. Just as for free surfaces, there may be altered grain boundary phases in the material as grown. In particular, under certain conditions, including one grain having a near [001] normal at the boundary, there can be 1:2:4 YBCO (i.e. $\mathrm{YBa}_{2} \mathrm{Cu}_{4} \mathrm{O}_{y}$ ), as opposed to the usual 1:2:3 phase (Kogure et al 1988). This phase is not seen when both grains (or neither of them) have a grain boundary normal near [001]. The formation of this phase at grain boundaries is affected by the conditions of oxygenation. There are several reports of amorphous regions at grain boundaries (e.g. the work of Ramesh et al (1990) on $\mathrm{Pb}-\mathrm{Sr}-\mathrm{Ca}-\mathrm{Er}-\mathrm{Cu}-\mathrm{O}$ ).

A comparison of Auger electron spectra from intergranular and transgranular areas exposed on fracture surfaces of YBCO indicates (Kroeger et al 1988) that most grain boundary surfaces are oxygen deficient and rich in copper compared to the bulk material. The modified region is estimated to be $15-50 \AA$ thick. No evidence of segregation of impurities to grain boundaries was seen in this study. The results indicate-in line with other data - that the grain-boundary layer is nonsuperconducting and a likely contributor to the low critical current densities in these materials. Possibly an additional factor is carbon at grain boundaries, which may result from incomplete calcination in material which is slightly off stoichiometry. Parallel results are found using high-spatial-resolution STEM in conjunction with energy dispersive $\mathrm{x}$-ray analysis and scanning Auger electron spectroscopy (Babcock et al 1988). The majority of grain boundaries in YBCO were found to be copper-rich and bariumand oxygen-deficient. A minority were found to contain a thin layer (about $1.5 \AA$ ) of copper-rich, but barium- and yttrium-deficient oxide material.

The grain size dependence of magnetic and resistive properties in $\mathrm{La}_{1.85} \mathrm{Sr}_{0.15} \mathrm{CuO}_{4}$ (Chiang et al 1988) suggest equilibrium segregation of $\mathrm{Sr}$ at the grain boundaries, not unlike that of $\mathrm{Ca}$ in $\mathrm{MgO}$. 
Many workers report layers with altered dielectric properties at grain boundaries (for example, Ginley et al 1987, Cohen et al 1987, Goldfarb et al 1987, Naito et al 1987). The main results are these. First (Ginley et al 1987), deoxygenation and reoxygenation does not affect the structure of the grain interconnects in the material. Secondly, (Cohen et al 1987) surface impedance data are found to be well described by an accepted model of an inhomogeneous material. Near the critical temperature, the material can be treated as an assembly of isolated superconducting grains embedded in a normal matrix of low conductivity dielectric, on a scale small compared to the skin depth (which is about $200 \mathrm{~nm}$ ). Electron tunnelling into thin films of superconducting La compound (Naito et al 1987) show an exceptionally large energy gap, larger than seen previously in bulk sintered powders; this is interpreted as due to large anisotropy in the material or to the presence of a superconductor with a very high critical temperature near the surface of the films.

4.2.3. Twin boundaries. Wen et al (1988) have identified seven types of twin boundary in YBCO by means of electron microscopy techniques. They remark that (i) the twinning provides pinning for a high critical current and (ii) that a high critical current is also aided by the continuity of $\mathrm{O} 1-\mathrm{Cu}-\mathrm{O} 1$ chains across twin boundaries. Grain boundaries with second phases are always obstacles to high critical currents; other types of grain boundary may be obstacles, with the exception of coherent grain boundaries (see for example Mueller et al 1988). A statistical analysis of microtwin domains in YBCO (Teodorescu et al 1989) indicates a periodicity in the distribution of the widths of the twin lamellae, the maximum periodicity being about $30 \mathrm{~nm}$. Such large-scale periodicities are known in conventional oxides, e.g. the ordering of shear planes (Stoneham and Durham 1973). There are also clear analogies with the Magneli series of YBCO compounds associated with the accommodation of non-stoichiometry by interstitial planes (Khachaturyan and Morris 1990).

Twins can apparently be removed from single crystal YBCO thermomechanically (Kaiser et al 1989), exploiting the ferroelastic behaviour of YBCO and other layered perovskites.

4.2.4. Subgrain boundaries. The examination of several dozens of grains (Verhoeven et al 1988) in an electron channelling study of YBCO shows that the majority of the large grains examined contained subgrains, misaligned by 0.5 to $1.0 \AA$, and ranging in size from less than $3 \mu \mathrm{m}$ to $20 \mu \mathrm{m}$. The origin of these subgrains was not understood.

\subsection{Oxide/metal contacts}

Even for simple oxides, the nature of their contact with a metal is only gradually becoming understood (Stoneham and Tasker 1989, Dufour and Nowotny 1989). The reasons for adhesion and coherence of contact will surely be similar in the superconductors, but experiments are still rudimentary in many respects. The influence of water on contacts shows many of the features expected from reaction with atmospheric moisture. Thus (Chang and Tsai 1988) the immersion of superconducting films of YBCO in water shows large differences in degradation between structures with and without silver. Water immersion at $20^{\circ} \mathrm{C}$ causes rapid degradation of $\mathrm{YBCO}$, affecting especially the contact resistance (which is poor after a mere $5 \mathrm{~min}$ ) and the normal state resistance; however the silver-containing material survives far better. 


\subsection{Substrates}

Substrates are required to provide a variety of functions: epitaxy to ensure optimum structure, lack of dielectric loss for microwave applications, and-a very important point-chemical inertness at all stages of operation. Some standard materials fail badly. As regards chemistry, Koinuma et al (1988) have investigated the solid phase chemical reaction between $\mathrm{YBCO}$ and substrate materials. This was done by $\mathrm{x}$-ray diffraction and $\mathrm{AC}$ susceptibility measurements. The $\mathrm{x}$-ray diffraction measurements imply that it is the barium in YBCO that is primarily responsible for the reactions with substrate materials, where it forms either barium fluoride or a complex oxide. The electronegativity of components of the substrate have a qualitative correlation with the reactivity of YBCO towards the substrate material. The order of reactivity is:

Most reactive:

(i) tungsten carbide; chromia; calcium fluoride;

(ii) silicon; amorphous silica;

(iii) Y-stabilized zirconia; SUS-316 stainless steel; zirconia; rutile; alumina.

Least reactive:

(iv) strontium titanate; MgO.

Strontium titanate's phase transition makes it unsuitable for microwave applications. Dechannelling studies ( $\mathrm{Li}$ et al 1989b) indicate the presence of stress-relief dislocations for $\mathrm{YBCO}$ on $\mathrm{MgO}$ substrates. $\mathrm{MgO}$ also appears to show negligible interdiffusion with BISCCO (Kuroda et al 1989). A sputtered film of BISCCO on a $\mathrm{MgO}$ substrate was observed to show superconductivity in an extremely thin state, with quite good critical temperatures ( $100 \mathrm{~K}$ onset and $65 \mathrm{~K}$ zero resistance), consistent with negligible interdiffusion. Other work (Fogarassy et al 1988) indicates little reactivity between the BISCCO compounds and substrates of yttria-stabilized zirconia.

Intermediate layers have proved an effective way of eliminating unwelcome reactions, as well as improving adhesion and quality of growth. Thus $\mathrm{MgO}, \mathrm{ZrO}_{2}$ and $\mathrm{Al}$ have all been used for Si substrates. The use of an Al barrier, for instance (Byrne et al 1989) improves adhesion and prevents the formation of $\mathrm{Cu}$ precipitates at the interface. The $\mathrm{Al}$ does not affect the presence of a $\mathrm{Ba} / \mathrm{Si}$ layer at the interface; the $\mathrm{Al}$ itself appears to percolate into the $\mathrm{YBCO}$ film. Al also reacts strongly with the $\mathrm{Bi}$ compounds, reducing the $\mathrm{Cu}$ valency even at the lowest coverages (Wells et al 1989).

\section{Solid state processes: I. Diffusion}

\subsection{Mechanism and control}

In diffusion we are concerned with issues of mechanism and of control. As regards the mechanism, we will wish to know which atomic jump processes are involved, their relative rates, and their dependence on control parameters like temperature or stoichiometry. By control we mean that we can adjust certain parameters or impose certain constraints at our disposal to obtain an optimum process condition or desired final state. For example, we may wish to obtain a large single crystal of a phase which is only stable at below a certain temperature; we may find that the standard ceramic processes based on diffusion of a component cannot be achieved in a realistic time, and so have to seek other strategies based on low-temperature routes. 


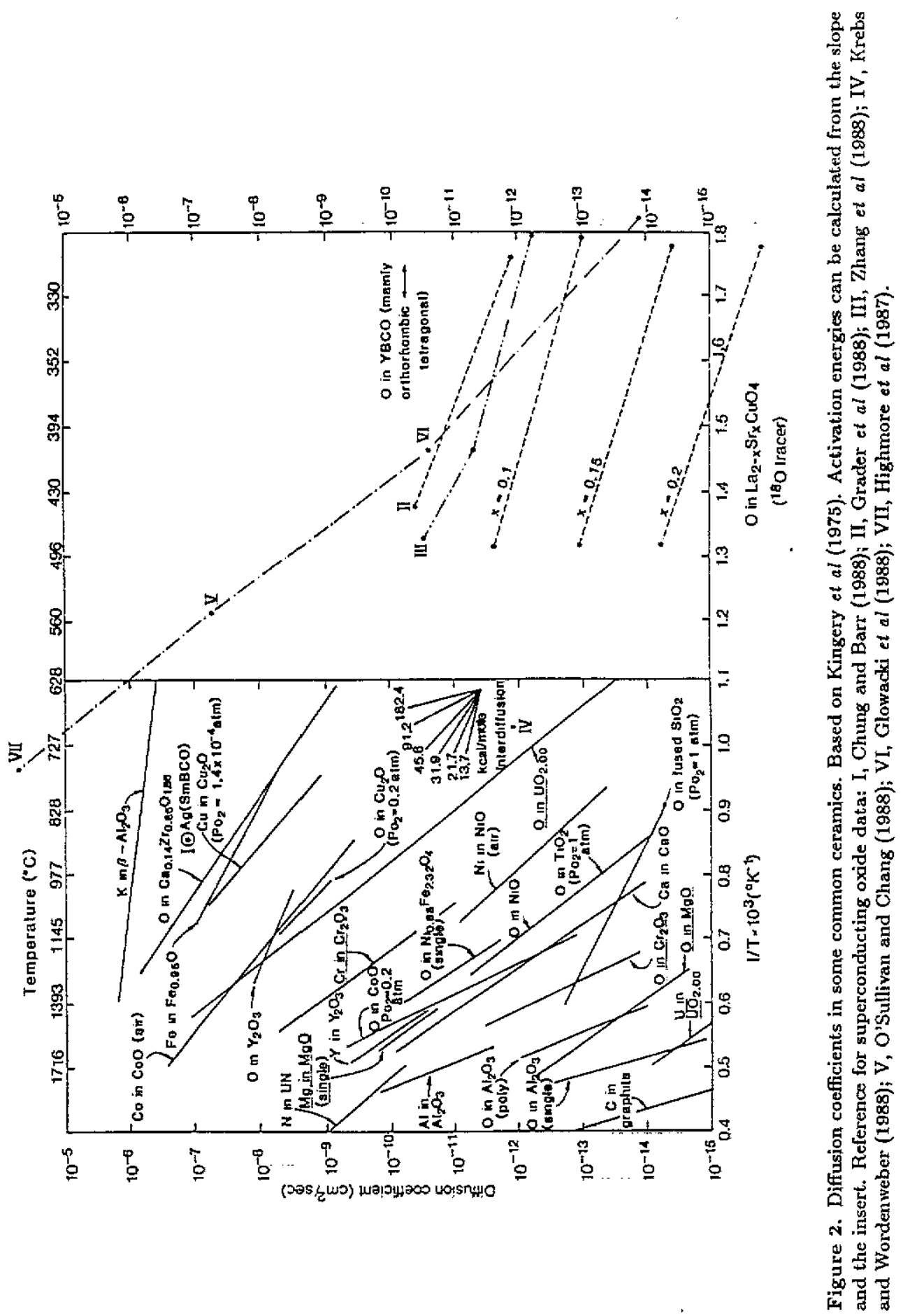




\subsection{Diffusion in non-superconducting oxides}

The diffusion processes in standard non-superconducting oxides form the basis of much of ceramic technology. Preparative processes like sintering and mechanical response like creep are good examples. In many cases for these processes it is the slowestmoving species which is rate determining, though for other features (e.g. obtaining a critical stoichiometry) the fast-moving component suffices. Figure 2 (based in part on Kingery et al 1976) shows a compilation of diffusion rates for a range of oxides. Some of the data should be taken with discretion, since there are frequently unintended impurity effects, especially for very slow diffusers.

We may make three main observations.

(i) Even the fastest diffusers have diffusion constants below $10^{-5} \mathrm{~cm}^{2} \mathrm{~s}^{-1}$. The superionic conductors ( $K$ in beta alumina; $O$ in stabilised zirconia, etc) show fastest diffusion, but well below what would result from jumps of length equal to the nearest neighbour spacing proceeding every lattice vibrational period.

(ii) Cuprous oxide is remarkable for having strikingly high diffusion rates for both anion and cation. Copper diffusion (not yet measured in superconducting oxides) is known to be fast in many systems, especially semiconductors, where it is a considerable nuisance.

(iii) Oxygen has a diffusion constant which can be strongly dependent on stoichiometry. Other sample to sample variations are to be found in granular versus single crystal samples, since grain boundary diffusion provides a fast path in many cases.

There have been many detailed computer simulations of diffusion mechanisms in oxides. Whilst some questions remain open for very non-stoichiometric oxides or for those with open-shell transition metals, the general agreement with experiment is very good. For instance, the diffusion rate (or strictly the $\mathrm{Mg}$ vacancy mobility) for $\mathrm{Mg}$ in $\mathrm{MgO}$ can be predicted about as well as it can be measured (for a review see Stoneham (1989b). As regards ternary oxides, far less work has been done. However, Lewis (1983a, p 146) achieves very respectable results for $\mathrm{BaTiO}_{3}$, with predicted activation energies in broad agreement with experiment:

$\begin{array}{lcl}\text { Vacancy } & & \begin{array}{l}\text { Experimentally } \\ \text { observed }\end{array} \\ \text { species } & \text { Predicted } & \text { Probably not seen } \\ \mathrm{Ti} & 15.12 \mathrm{eV} & 2.76 \mathrm{eV}(?) \\ \mathrm{Ba} & 3.45 \mathrm{eV} & 0.45-0.68 \mathrm{eV}, 0.55 \mathrm{eV} \\ \mathrm{O} & 0.62 \mathrm{eV} & 0.00\end{array}$

There is also a process reported with a $2 \mathrm{eV}$ activation energy, but its identity is uncertain.

\subsection{Types of diffusion constant}

Some care is needed in relating to each other diffusion constants observed in different ways in non-stoichiometric solids. In an atomistic model, what is calculated will usually be a jump rate, i.e. the probability per unit time of a single diffusive jump of the species which is rate determining. In an experiment, what is usually measured is a diffusion flux or perhaps a concentration profile. In an NMR experiment, however, where a jump rate is obtained, it can happen that long-range diffusion is not involved, 
and that the characteristic time observed is merely some local motion. Thus in the perturbed angular correlation studies of Indium at (probably) $\mathrm{Cu}(1)$ sites in $\mathrm{YBCO}$, in which the very low activation energy $((14 \pm 4) \mathrm{meV})$ is attributed to oxygen motion (Singh et al 1989b), one assumes this is a local activated process, as the energy is far smaller than from other experiments.

For a given diffusing species we can define three useful diffusion constants, namely (i) $D$, the random walk coefficient, (ii) $D^{*}$, the tracer diffusion coefficient, and (iii) $D^{\prime \prime}$, the partial chemical coefficient of diffusion. For given conditions, these three constants must all imply the same flux and same ionic jump rates, if properly interpreted. The links between these several diffusion constants are as follows:

Fick's law: $\quad J=-D^{\prime \prime}(\mathrm{d} c / \mathrm{d} z)$

where $D^{\prime \prime}$ is the chemical coefficient of diffusion and $J$ the flux

$$
J=-(D c / k T)(\mathrm{d} \mu / \mathrm{d} z)
$$

where $D$ is the random walk coefficient and $\mu$ the chemical potential.

$$
D^{\prime \prime}=D(1+\mathrm{d} \ln g / \mathrm{d} \ln c)
$$

where $\mathrm{g}$ is the activity coefficient.

The tracer diffusion coeffient $D^{*}$ is $f D$, where $f$ is the correlation factor.

One result is that those workers who measure, in effect, a chemical diffusion constant $D^{\prime \prime}$ (including those who monitor the orthorhombic/tetragonal transition as the oxygen concentration is changed by altering the external oxygen pressure) will get very different values from those who measure tracer diffusion coefficients. The linking equation is (for oxides, where the gas molecule is diatomic)

$$
D^{\prime \prime}=\left(D^{*} / 2 k T\right)\left(\mathrm{d} \delta G\left(\mathrm{O}_{2}\right) / \mathrm{d} \ln c\right)=\left(D^{*} / 2 k T\right)\left(\mathrm{d} \ln p\left(\mathrm{O}_{2}\right) / \mathrm{d} \ln x\right) .
$$

Thus the rapid variation of equilibrium oxygen stoichiometry $\mathrm{x}$ with external oxygen pressure is the reason for the large difference between $D^{\prime \prime}$ and $D^{*}$ in YBCO.

\subsection{Diffusion in $\mathrm{YBa}_{2} \mathrm{Cu}_{3} \mathrm{O}_{7-x}$}

It must be said that the existing diffusion data for oxide superconductors do not yet form a coherent picture in detail. There are gross differences from group to group, and even those who have a wealth of experience in diffusion measurements have not wholly resolved the problems. Nevertheless, some features are clear.

5.4.1. Oxygen diffusion. For Oxygen diffusion there have been at least twenty papers for YBCO. These show the following main features:

(i) the oxygen mobility is reported to be highly anisotropic, being fast along the $c$ axis (Couach et al 1988).

(ii) Some experiments measure chemical diffusion, and may well involve distinct processes from those which are based on other phenomena, like oxidation or reduction. Certain of the reported diffusion rates are very high, although those where the values are more extreme are not direct measurements. 
There is a significant dependence of activation energy on stoichiometry, at least in that the tetragonal phase has lower values than the orthorhombic phase. Internal friction data (Xie et al 1989) show diffusional jumps within the basal plane of YBCO which occur between sites $A\left(\frac{1}{2}, 0,0\right)$ and $\mathrm{B}\left(0, \frac{1}{2}, 0\right)$ which are not crystallographically equivalent in the orthorhombic form. Diffusion in the basal plane shows a pre-exponential factor and one component of the activation energy to be essentially independent of temperature between $400^{\circ} \mathrm{C}$ and $650^{\circ} \mathrm{C}$, this energy corresponding to that for the internal friction peak seen at $200^{\circ} \mathrm{C}$. However, the second part of the activation energy, related to the difference in energy between the $A$ and $B$ sites, does change with temperature, falling from $0.23 \mathrm{eV}$ at $400^{\circ} \mathrm{C}$ to zero at $670^{\circ} \mathrm{C}$, the temperature of the orthorhombic-tetragonal transition, when the sites become equivalent.

Some experiments exploit the fact that the orthorhombic phase nucleates at a grain boundary and then develops by volume diffusion (however, some assumptions are made here; also the reversibility of the process depends on the oxygen pressure). From observation of the orthorhombic/tetragonal phase transition kinetics, Arrhenius energies for the orthorhombic phase are found to be in the range 0.8 to $1.4 \mathrm{eV}$, consistent with many other 'superionic' oxides.

In order to see the effect of oxygen diffusion on the tetragonal-orthorhombic phase transformation, Y K Park et al (1988) interrupted oxygen diffusion by rapid cooling and then observed the microstructure of the specimens under cross-polarized light using an optical microscope. It was possible to see the path of oxygen diffusion by observing the variation of the concentration of twins, which are observed to form in the tetragonal-orthorhombic phase transition in $\mathrm{YBCO}$. At the boundary between the transformed and untransformed materials, evidence for diffusion-driven transformation could be clearly seen. This showed that oxygen diffuses through the bulk of the tetragonal grains more slowly than through grain boundaries and pores. Twinned areas were seen to be elongated in the direction of the long-grain axis; this may be a possible indication of anisotropy of oxygen diffusion in the YBCO system. Shi et al (1989) also used polarized light microscopy and were able to directly observe the oxygen diffusion fronts in YBCO samples through the orthorhombic-tetragonal transition. They found that the transition is directly controlled by oxygen diffusion and that the oxygen concentration of 6.5 is a sharp boundary separating the two phases.

(iii) Tracer data yield higher activation energy values, about $1.8 \mathrm{eV}$, although these results (Ikuma et al 1988) were obtained at lower temperatures. The prefactor was normal (about $0.03 \mathrm{~cm}^{2} \mathrm{~s}^{-1}$ ).

(iv) As regards processing (see Yan et al 1988) for process related problems), there is a clear asymmetry between oxygen removal and oxygen addition. Clearly, there is some difference from the different rates in the orthorhombic and tetragonal materials. But also $\mathrm{Tu}$ and his colleagues (Tu et al 1988) have reported that out-diffusion (oxygen removal) is limited by a surface reaction with a characteristic energy of $1.7 \mathrm{eV}$. This is not noted by others (e.g. O'Sullivan et al 1988), but not all experiments or samples are comparable. The result of the asymmetry is that more samples obtained by outdiffusion have more uniform oxygen levels, and less uniform samples are obtained by indiffusion, where an outer shell develops with a higher activation energy $(1.3 \mathrm{eV})$. This has clear implications for preparation routes.

Activation energies quoted for the diffusion of oxygen into $\mathrm{YBa}_{2} \mathrm{Cu}_{3} \mathrm{O}_{x}$ show dependence of the activation energy on $\mathrm{x}$, with values of $1.1 \mathrm{eV}$ for $x=7$ and $0.38-0.48 \mathrm{eV}$ for $x=6.62$ 
(v) The activation energy for oxidation or reduction (J-H Park et al 1988) is only $0.3 \mathrm{eV}$; again the interpretation needs caution because of a substantial disagreement with other work, perhaps associated with the effects of shell structure on the grains.

(vi) Note that, as for some conventional oxidation processes, e.g. the oxidation of silicon, it is not always possible to distinguish between reaction-limited (here there could be some local reorganization unrelated to the overall composition gradient) and diffusion-limited behaviour in all circumstances.

In YBCO, there is a direct correlation between oxygen deficiency and ordering in the copper oxide planes and the lowering of the superconducting transition temperature. In order to study the chemical nature of these $\mathrm{Cu}-\mathrm{O}$ bonds, $\mathrm{Li}$ et al (1989a) did a detailed study of the copper oxides. Using thin film samples, they oxidized $\mathrm{Cu}_{2} \mathrm{O}$ into $\mathrm{CuO}$ and reduced $\mathrm{CuO}$ back to $\mathrm{Cu}_{2} \mathrm{O}$. During the reduction, they observed an abrupt change in the oxygen concentration across the phase boundary between $\mathrm{CuO}$ and $\mathrm{Cu}_{2} \mathrm{O}$. This behaviour is quite different from that seen in the oxidation and reduction of $\mathrm{YBCO}$, where the change in oxygen concentration is continuous. They propose that this discontinuous morphology of grain growth of $\mathrm{Cu}_{2} \mathrm{O}$ is due to the migration of the $\mathrm{Cu}_{2} \mathrm{O}-\mathrm{CuO}$ phase boundary induced by oxygen out-diffusion along the moving phase boundary.

Song et al (1990) monitored the electrical resistivity of single phase polycrystalline YBCO samples while changing their oxygen content in both ozone and ordinary oxygen environments and thus were able to investigate the correlation between their average oxygen content and the diffusion time for oxygen inside grains. They concluded that while ozone and ordinary oxygen may have different effects in oxidizing the surface of grains, they show no observable differences in the oxygen diffusion process in the bulk YBCO material.

5.4.2. Metal diffusion. (i) For cation diffusion there is a single measurement, namely $\mathrm{Ag}$ in $\mathrm{SmBCO}$. This shows fast diffusion (about $10^{-7} \mathrm{~cm}^{2} \mathrm{~s}^{-1}$ at $931^{\circ} \mathrm{C}$ ) though this could, presumably, be grain boundary diffusion, both because the sample had $25 \%$ porosity and because reports of $\mathrm{Ag}$ indicate it remains associated with grain boundaries (Chung et al 1988). The high temperature $\left(870-980^{\circ} \mathrm{C}\right)$ deformation of YBCO shows a stress exponent close to 1 (von Stumberg et al 1989), consistent with diffusion as the main deformation mechanism. Here the slowest-moving species (presumably metal motion, probably along grain boundaries) is rate-determining; the activation energies reported are large $(6-8 \mathrm{eV}$, decreasing as the oxygen pressure rises). The flow stress varies roughly as the inverse square root of the oxygen pressure.

(ii) Metal diffusion from substrates or contacts has been remarked on already. Rutherford backscatter (Nakajima et al 1988) has been used to look at the diffusivity of $\mathrm{Cu}$ in $\mathrm{MgO}$, alumina, quartz, and $\mathrm{Si}$ substrates (where $\mathrm{Cu}$ moves faster than $\mathrm{Y}$ or $\mathrm{Ba}$ ) and also to study the motion of substrate elements $(\mathrm{Mg}, \mathrm{Si}, \mathrm{Al})$ in $\mathrm{YBCO}$. They concluded that $\mathrm{MgO}$ seems the most stable, although other workers (Sugita et al 1988) suggest that small $\mathrm{Mg} / \mathrm{Cu}$ interchange from the $\mathrm{MgO}$ substrate during annealing leads to a reduction in critical temperature; their samples showed variable range hopping corresponding to 0.5 states $\mathrm{eV}^{-1} /$ cell below $100 \mathrm{~K}$. Auger depth profiling has also been exploited to look for interdiffusion between a thin superconducting film of YBCO and the $\mathrm{MgO}$ substrate (Ma et al 1988). One would expect that a decrease in the synthesis temperature for thin films would reduce the interdiffusion between the film and the substrate, and this is observed (Wasa et al 1988). 


\subsection{Diffusion in the La compound $\mathrm{La}_{2-x} \mathrm{Sr}_{x} \mathrm{CuO}_{4-y}$}

Careful isotope tracer work has been performed for oxygen diffusion in the La compound $\mathrm{La}_{2-x} \mathrm{Sr}_{x} \mathrm{CuO}_{4-y}$ as a function of $\mathrm{Sr}$ content (Routbort et al 1988). From this one learns two special points of interest:

(i) there is an increase in activation energy as the $\mathrm{Sr}$ concentration rises (77 kJ mol ${ }^{-1}$ for $x=0.1$ and $108 \mathrm{~kJ} \mathrm{~mol}^{-1}$ for $x=0.2$ ).

(ii) there is a prefactor change with stoichiometry. The changes can only be understood simply if the oxygen vacancies become bound to strontium clusters and thus immobilised. This is in contrast with what one might have expected, namely a diffusion coefficient increase with increasing vacancy concentration caused by the added strontium. The diffusion constant decrease with increasing strontium arises mainly because the activation energy increases.

\subsection{Further aspects of diffusion}

5.6.1. The reactive element effect. In the growth of oxide films, diffusion is often dominated by grain boundary paths. In certain cases, very small additions of key dopants have a profound effect on the rate, which is strongly suppressed. A general review of the reactive element effect has been given by Polman et al (1989).

The solid-state science of complicated diffusion-controlled oxide growth of chromium and chromia-forming alloys is relatively well-understood, both theoretically and for systematic application. Additions of doping elements (the so-called 'reactive elements') such as cerium, yttrium, lanthanum etc to chromia-forming alloys has a very beneficial effect in reducing the growth rate of oxide films, an effect also noted for ionic transport processes in related crystal, e.g. yttrium in $\mathrm{Cr}_{2} \mathrm{O}_{3}$. STEM measurements give experimental support for a model of grain-boundary segregation and some mechanism of blocking of grain boundary diffusion by the reactive elements. The reactive element effect may involve physical blocking of the lowest-energy diffusion pathways but it is not clear that the solubility of reactive elements is high enough to explain the observed reduction of the oxidation rate by this model. Other mechanisms involve the formation of new phases at critical parts of the boundaries, or indeed a modification of the nature of the microstructure. Whatever the process, its effect can be major. For oxide superconductors, the phenomenon has not been remarked on, though there are significant effects of dopants in modest concentrations; we have already commented on the effects of silver. Given that the superconducting behaviour is sensitive to grain/grain character as well as to oxygen level, it is highly likely that similar 'reactive element' phenomena will be found in these oxides, and that these will affect processing.

5.6.2. Sintering. The most common applications of surface diffusion occur in the processing of solids with very high melting points. Most metals can be cast or machined; most semiconductors can be grown from the melt or by molecular-beam epitaxy. However, ceramics are most easily obtained as powders or similar aggregates. The aggregated forms are consolidated by heat treatments such as sintering and hot pressing, which may exploit surface diffusion. The processing of high-melting-point oxides or other ceramics is aimed at controlling specific physical features, e.g. the porosity. The porosity affects apparent bulk diffusion. Another feature concerns grain sizes and compositions. There are three main mechanisms of surface diffusion; a vacancy mechanism (exactly as in bulk diffusion); the motion of atoms or ions along 
the surface (adatoms) in contact with the outer layer of surface atoms; and thirdly at very high temperatures there can be vapour transport. The lower activation energy of grain-boundary diffusion means this will al ways dominate over bulk diffusion at lower temperatures. So in low-temperature oxidation, it is the grain boundaries that limit the rate of oxidation. High angle boundaries are especially important. In the main consolidation occurs through the infilling of the narrow neck regions near points of contact of the grains. The several different mechanisms are classified by the source (e.g. other surface regions, grain boundaries or dislocations) and the type of diffusion involved (surface, bulk or grain-boundary). In different regimes of temperature and geometry, the sintering is dominated by different mechanisms. For further discussion see Kingery et al (1976) and Hayes and Stoneham (1985).

5.6.3. Enhanced diffusion. In many experiments it is found that the measured values of the diffusion constant are far higher than that expected for the perfect bulk material. This is due to the existence of extended defects or defective regions in the material, which can act as easy paths for atom migration. For polycrystalline materials, diffusion along grain boundaries certainly predominates in most diffusion. Theoretical models have been developed to take into account the role of dislocations. The effective diffusion constant can be written as the sum of a bulk lattice term and a term proportional to the dislocation length per unit volume. Other forms of enhancement are possible in the presence of other phases which affect point defect concentrations locally, as in the effect of alumina inclusions on $\mathrm{Li}$ diffusion in LiI. There is certainly scope for such effects in the superconducting oxides that are not single-phase, but there are no specific reports yet.

\section{Solid state processes: $\Pi$. Radiation damage and amorphization}

The broad features of radiation damage in oxides have been obtained mainly from experiments on binary oxides and, even there, much use has been made of analogies with the alkali halides. The studies of ternary oxides have normally been concerned with preserving insulation for fusion applications, so that the emphasis has been on structural integrity and on radiation-enhanced conduction. One important problem for oxide superconductors is that, even above the critical temperature, one does not understand either the radiation response or the dependence on temperature of the resistance. In particular, it is not understood why the resistance $R$ shows changes in $\mathrm{d} R / \mathrm{d} T$ which are roughly linear in the fluence $\Phi$. There is a clear need for studies of normal oxides which are similar in structure to the superconducting ones.

\subsection{General features: types of damage}

In all solids, one expects the purely collisional effects in radiation damage to follow some broad rules. The small electron mass means that (in the absence of any specifically electronic effects) only very high energy electrons can cause displacement; since the secondaries will have only small energies, one expects well-separated point defects. For protons, the glancing collisions which dominate Rutherford scattering transfer relatively little momentum, so only a few secondaries per primary are anticipated. In contrast, the hard-sphere interactions of neutrons lead to large energy transfers, and compact, highly damaged regions are expected. Heavy ions and fission fragments may leave heavily damaged tracks, possibly $10 \mu \mathrm{m}$ long, as they deposit their energy. 
The characteristic energy for the effects of collisions is the displacement energy, i.e. the energy transfer needed to cause ion displacement. Data for simple oxides include the following:

$\begin{array}{lll}\text { Cation displacement } & \begin{array}{l}\text { Oxygen displacement } \\ (\mathrm{eV})\end{array} \\ \mathrm{MgO} & 52 & 54 \\ \mathrm{CaO} & 50 & 50 \\ \mathrm{Al}_{2} \mathrm{O}_{3} & 18 & 75 \\ \mathrm{ZnO} & 30-60 & 60-120\end{array}$

These data suggest that the cation is more readily displaced. Such a simple view is not general-electron irradiation of lithium niobate (Hodgson et al 1989) or lithium aluminate (Auvray-Gely 1989) appears to give oxygen vacancy centres-and is complicated by the effects of ionization, for there are electronic mechanisms of defect production and movement too. It is worth remarking that the analogue of the displacement energy for electronic excitation is the generation of an electron-hole pair, since this is the source of secondary displacements by electronic means. When produced by high-energy sources, like x-rays, electron hole pair generation needs about three times the energy gap (Alig et al 1975). When low energy sources are used, mere band gap excitation suffices in several cases (Stoneham 1989c).

For the superconducting oxides, it is almost certain that it is oxygen which is displaced most easily, and working values for the displacement energy are about $20 \mathrm{eV}$. On this basis, most workers report:

$$
\begin{aligned}
& \text { Damage affects superconductivity }>\text { a few } x 0.01 \mathrm{dpa} \\
& \text { Damage destroys superconduction }>\text { about } 0.05 \mathrm{dpa} \\
& \text { Damage amorphizes the material }>1-2 \mathrm{dpa}
\end{aligned}
$$

where dpa means the estimated average number of displacements per atom.

\subsection{Radiation damage in ionic solids}

Non-metallic compounds exhibit different damage behavjour from the relative simplicity of metals. How do these features emerge in oxide superconductors, which are both metals (albeit sometimes bad metals or even semiconductors) and oxides? The special features of compounds like the ionic oxides are the following.

(i) The defect species on the different sublattices are quite different (so anion vacancy defects are distinct from cation vacancy defects in clearly observable ways). This difference is deeper than that from host crystal structure alone (for anion vacancies in various oxides have similarities) or from recognising the importance of ion mass in energy transfer. This feature continues to be true for the superconductors, where the special role of oxide stoichiometry matters, and where the $\mathrm{Cu}$ also has an individual contribution.

There is strong evidence for the oxide superconductors that-to a first approximation-only damage on the oxygen sublattice results from any sort of irradiation. This follows from (a) the fact that the radiation effects seem to occur systematically at specific dpa (this would not be so if different sublattices were responding differently to different radiations) and (b) from the striking 'universal' behaviour whereby (over seven orders of magnitude) the value of $\mathrm{d}$ (reduction in $T_{\mathrm{c}}$ )/ $\mathrm{d} \Phi$ 
values are linear in nuclear energy deposition. This does not mean that only oxygen is displaced-clearly that would be wrong in heavy-ion induced cascades-but that the cation sublattices recover to a great extent. But the universal behaviour does make credible models of the 'shake everything up' sort; this could, perhaps, have been anticipated from the way that complex glasses used for incorporating radioactive waste appear to move systematically to an 'ideal' density whose value is independent of the activity (Summers et al 1989).

(ii) In ionic oxides, charged defects can occur. Charge compensation, charge conservation and the long-range Coulomb interaction are of major importance. The message is not too clear in oxide superconductors. Obviously there are not well-defined ions in the same sense as in ionic solids (or even in semiconductors (Catlow and Stoneham 1983) yet (a) there may be insulating phases or regions which are ionic (perhaps at grain boundaries), (b) 'charge compensation' rules do seem to help in understanding many phenomena, (c) the anisotropy of screening may allow some value in using symbols like ' $\mathrm{Cu}^{3+}$ '; a related question concerns the range of electronic densities over which molecular binding can be an acceptable description, e.g. in species like peroxy, and (d) data for some temperature ranges show variable range hopping in a form more appropriate for an insulator. Thus the possibility of several distinct charge states for defects introduced by radiation should not be dismissed too quickly, even for these metallic oxides.

(iii) Damage initiated on one sublattice tends to stay on that sublattice. This is extremely important in binary solids, and results from several causes: (a) focussed collision sequences tend to occur only along rows of a single species (a factor unlikely to be critical in the complex structures of oxide superconductors); (b) diffusion processes may involve only a single ion; (c) Madelung terms (or their analogues) tend to prevent species $\mathrm{D}$ occupying a site of species $\mathrm{E}$.

Even if the description in terms of Coulomb interionic interactions is not wholly appropriate in metals, strong site selectivity is found for substitutions in the new oxide superconductors. Moving species from one site type to another involves a defect form which, in effect, is a low energy antisite defect. In alkali halides this is a halogen molecule in an anion-cation divacancy; in III-V semiconductors, the simple antisites are low in energy. In ternary oxides one expects that cation sublattices could be intermixed, or that oxygen molecules in neutral vacancy complexes would be able to help transfer damage from one sublattice to another. Such site selectivity is implied in the 'reference crystal' model described previously.

The need for transfer of damage from one sublattice to another comes if one wants to explain dislocation climb, or the existence of perfect dislocation loops or the appearance of large void regions. In BISCCO there is clear evidence of cation reorganization too from the formation of perovskite phases. The changes in structure described as 'unzipping' of the basal plane are also evidence for $\mathrm{Cu}$ reorganization. There is also indirect evidence, such as the fact that an unirradiated material with a $60 \mathrm{~K}$ critical temperature is not equivalent in its radiation response to $90 \mathrm{~K}$ material degraded by irradiation (the rule is that poor material damages more readily).

(iv) In many ionics there are excitonic mechanisms of defect generation. In the alkali halides, the sequence is (a) free exciton formation; (b) exciton self-trapping as the hole yields a molecular halogen ion to which the electron is bound; (c) the nonradiative decay of the self-trapped exciton (with its [110] axis) has a channel which leads to a collision sequence along the close-packed [110] direction, producing a distant interstitial and leaving a vacancy. The anion defect species is dominant, though there 
is also evidence for a small component of cation defect production by a subsidiary mechanism. In alkaline earth fluorides, the process is inhibited because the axis of the self-trapped exciton does not correspond to that to the close-packed row.

In ionic oxides, the steps must be slightly different, because the (divalent) oxygens cannot form a stable molecule by trapping just one hole. Instead, what seems to happen (as a reflection of the importance of the Coulomb terms) is that, in effect, an oxygen atom is moved to an interstitial site. There is therefore a transient oxygen interstitial/vacancy pair. Whether this leads to longer-term damage depends on other factors. In oxide superconductors, there are reports of exciton-like transitions, but it is far from clear that these are in the same phases as the superconduction. However, if peroxy formation is real, or if molecular oxygen interstitials are indeed formed, then it is possible that even short-lived electronic excitation (including core excitation) could generate damage.

\subsection{Amorphization}

There are at least six different forms of disordered oxides which are referred to as 'amorphous'. Since these are not necessarily the same in structure, and since the operational definition of amorphous is primitive (e.g. diffraction rings, not spots, or perhaps merely bad contrast in an electron microscope) the concept should be used with care. The six forms are (i) liquid, (ii) vitreous (no latent heat; no clear phase transition), (iii) splat-cooled/rapid quenched (a broader range than vitreous, and sometimes without the need for the standard network formers), (iv) the naturally amorphous oxides which form continuous random networks even when stoichiometric and pure, like silica, $(v)$ the metamict form, in which radiation damage leaves the external habit unaltered whilst making the optical response isotropic, and (vi) ion beam amorphized. Under irradiation, Naguib and Kelly (1975) define various responses, which may be grouped as follows:

$\begin{array}{llll}\text { Initial Quenches } & \text { Quenches to } & \text { Quenches } \\ \text { form } & \text { to crystal } & \text { glass if alloyed } & \text { to glass }\end{array}$

Resulting form

Lower oxide

Metamict

Amorphous

glass if alloyed

to glass

$$
\begin{aligned}
& \mathrm{WO}_{3}, \mathrm{MoO}_{3} \\
& \mathrm{SiO}_{2} ; \mathrm{GeO}_{2}
\end{aligned}
$$$$
\mathrm{Al}_{2} \mathrm{O}_{3} ; \mathrm{TiO}_{2} \quad \mathrm{Fe}_{2} \mathrm{O}_{3} ; \mathrm{TiO}_{2}
$$$$
\mathrm{Nb}_{2} \mathrm{O}_{5} ; \mathrm{Ta}_{2} \mathrm{O}_{5} \quad \mathrm{Nb}_{2} \mathrm{O}_{5}
$$$$
\mathrm{Bi}_{2} \mathrm{O}_{3} ; \mathrm{Cr}_{2} \mathrm{O}_{3}
$$$$
\mathrm{SnO}_{2}
$$

It has yet to be identified which form results from the irradiation of oxide superconductors. However, since some of the apparently amorphous regions are parts of high angle grain boundaries (tilts above $13^{\circ}$ in YBCO), for which one might suspect merely complex structure. Yet radiation amorphization appears to be initiated at grain boundaries, so there may be a link to vitreous or similar nature. Displacement of the rare earth seems crucial. Many theories of superconductivity apply equally well to amorphous oxides, and it will be interesting to see if glassy superconductors do arise.

Clark et al (1987a) have examined the microstructure of ion-implanted thin films of YBCO by TEM. The superconducting properties of the films were dominated by large pancake-shaped grains of $\mathrm{YBCO}$ with their $c$ axis perpendicular to the substrate. 
Other grains of $\mathrm{YBCO}$ whose $c$ axis was parallel to the substrate formed spherulites. Irradiation with $500 \mathrm{keV} \mathrm{O}^{+}$ions caused amorphous zones to appear on the grain boundaries between the pancake grains, which initially were free of amorphous or second phases. At a higher dose $\left(2 \times 10^{14}\right.$ ions $\left.\mathrm{cm}^{-2}\right)$ a continuous amorphous layer was formed $150 \AA$ thick. However the interior of the grains showed no irradiationinduced microstructural features until they became amorphous at an even higher dose $\left(3 \times 10^{14}\right.$ ions $\left.\mathrm{cm}^{-2}\right)$. The appearance of the amorphous layer on the grain boundaries at low doses accounts for the reduction in $T_{c}$ observed in these films. Naguib and Kelly considered the criteria for bombardment-induced structural changes in non-metallic solids; there can be amorphization, crystallization or stoichiometry changes; they found empirically that amorphization should occur whenever the ratio (crystallization temperature) $/$ (melting point) exceeds 0.3 or the ionicity is greater than or equal to 0.47 . Anisotropic substances (where the term anisotropic can be understood to refer to bond directionality) tend to amorphize under ion impact, whereas cubic substances e.g. $\mathrm{NaCl}$, tend to remain crystalline. Naguib and Kelly show tables (part of which is given above) indicating which non-metallic solids amorphize on ion impact.

Egner et al (1987) studied the effects of irradiation-induced defects on the superconductivity of YBCO. The YBCO was irradiated with $\mathrm{He}, \mathrm{Ar}$ and $\mathrm{H}$ ions. Independent of the mass or energy of the ions, the reduction of critical temperature below $T=4.2 \mathrm{~K}$ occured in the region of $2 \times 10^{-3}$ to $1.6 \times 10^{-2}$ displacements per atom (dpa). The orthorhombic to tetragonal phase transition occured between 0.01 and $0.03 \mathrm{dpa}$, while the transformation to the amorphous phase was observed at about $0.1 \mathrm{dpa}$ after He irradiation at $77 \mathrm{~K}$.

\subsection{Summary of the main features observed}

6.4.1. Optical processes. Here a rich variety of behaviour is seen (including both conversion from and to the superconducting state), as well as oxidation and reduction, namely:

(i) precursors can be converted into superconductors by laser excitation (Gupta and Koren 1988), Liberts et al 1988b). Liberts et al increased the oxygen content of YBCO locally by laser-induced heating under $\mathrm{CW}$ Ar and Kr laser irradiation in an oxygen atmosphere.

(ii) Species may be ablated (Deshmukh et al 1988, Auciello et al 1988a,b, Becker and Pallix 1988). In their earlier paper (1988a), Auciello et al analysed a laser impacted area of YBCO. Scanning Auger microscopy revealed a significant depletion of $\mathrm{Cu}$ and spatial redistribution of $\mathrm{Y}, \mathrm{Ba}, \mathrm{Cu}$ and $\mathrm{O}$ on the surface. $\mathrm{X}$-ray diffractometry revealed a new broad peak characteristic of $\mathrm{BaY}_{2} \mathrm{O}_{4}$ and a poorly defined peak that can be attributed to $\mathrm{BaCuO}_{2}$.

(iii) Metallic tracks may be defined using a laser (Mannhart et al 1988, Liberts et al 1988a, Humphreys et al 1989). Liberts et al diminished the oxygen content of $\mathrm{YBCO}$ by local heating under $\mathrm{CW} \mathrm{Kr}$ laser irradiation in a hydrogen atmosphere. Clearly, laser methods allow the superconducting properties of material to be locally changed or destroyed.

The desorption behaviour has features of interest too: the excitation from specific core levels gives thresholds in behaviour (Rosenberg et al 1988). The first observations demonstrated synchrotron radiation could induce desorption of neutral oxygen molecules from a surface by core-level excitation. The observed thresholds correspond 
to excitation of $\mathrm{Cu}(3 \mathrm{p})$ and $\mathrm{Ba}(4 \mathrm{~d})$ core levels. The production of molecular oxygen at the $\mathrm{Cu}(3 \mathrm{p})$ edge involves excitation of shake-up or electron correlation states, while the desorption at the $\mathrm{Ba}(4 \mathrm{~d})$ edge appears to involve excitation of localized $\mathrm{Ba}^{2+}$ states ( $4 \mathrm{~d}$ to $4 \mathrm{f}, 5 \mathrm{f}$ transitions). Becker and Pallix (1988) have performed mass spectral investigations of ions produced directly from $1064 \mathrm{~nm}$ plus $532 \mathrm{~nm}$ laser-beam ablation of bulk superconducting YBCO. Cluster mass patterns ranging above 104 amu were observed. These were believed to be products of condensation rather than direct emission. Data for species in the ablation plume of YBCO and the Bi compound are given by Saenger (1989).

There are some analogies of laser ablation with laser annealing; for example, there is a critical laser flux for ablation. Kullmer and Bauerle 1988) find that for ablation of BISCCO by $\mathrm{XeCl}$ excimer laser in both air and vacuum, etching commences at about $2.4 \mathrm{~J} \mathrm{~cm}^{-2}$. There is once more the question of whether there are special compounds or structures which might be created by use of lasers to avoid processing temperatures above the stability limit of a useful metastable oxide, rather like metastable alloy formation by ion implantation, or high doping with laser anneal).

6.4.2. Gamma irradiation. Gamma irradiation seems to have relatively little effect on the superconductivity of ceramics. Boiko et al (Boiko et al 1988) report a slight improvement in superconducting properties, as do a Russian group (Babakishvili et al 1989); Vasek et al (Vasek et al 1989) report a slow degradation of properties; still others (Kutsukake et al 1989), Bohandy et al 1987) report virtually no effect at all. The main effects expected would be from the 'gamma wind' from the Compton effect, and this would matter only in regions of low electrical conductivity.

6.4.3. Electron irradiation. Electron beams offer a convenient way of modifying (usually degrading) superconducting behaviour, with potential for microelectronics applications. Whilst there are some observations of unstable radiation-induced increases in critical temperature, most workers find little effect (Bohandy et al 1987) or a degradation of performance. This degradation comes from two mechanisms:

(i) Amorphization (Nastasi et al 1988). This appears to be dependent on displacements occuring at the rare-earth lattice site ( $Y / G d)$, with faster amorphization noted at the grain boundaries;

(ii) Pair breaking, apparently by a magnetic mechanism (Hofmann et al 1988) which shows up as a correlation between the Curie constant and $T_{\mathrm{c}}$ during annealing, following irradiation with a dose of $4 \times 10^{19} \mathrm{~cm}^{-2}$. Non-irradiated stoichiometric samples have a nearly temperature independent paramagnetic susceptibility between 100 and $300^{\circ} \mathrm{C}$.

There are also several indirect effects of electron beams. If there is an insulating layer, then there should be a change in the way adsorbed oxygen molecules are dissociated and removed because of the classical electrostatic image effect. In essence, a thick film of insulator favours dissociative attachment of electrons $\left(\mathrm{O}_{2}\right.$ to $\mathrm{O}^{-}$and $\left.\mathrm{O}\right)$ whereas a thin film of insulator favours the production of two charged species (Sambe et al 1987). The dissociation of oxygen molecules by low-energy electrons may affect absorption kinetics too, as for silicon oxidation.

6.4.4. Neutron irradiation. The behaviour of the superconducting oxides under neutron irradiation is important for possible applications such as the design of magnets in the fusion reactor. 
The critical magnetization current may be improved in some cases by fast neutron irradiation, presumably by flux line pinning (Umezawa et al 1987, Wisniewski et al 1988, van Dover et al 1989). Umezawa et al found that neutron irradiation up to a fluence of $8.16 \times 10^{17} \mathrm{~cm}^{-2}$ systematically enhances the magnitude and reduces the anisotropy of the critical magnetization current compared to that of unirradiated samples (see also Wisniewski et al 1988), although most workers report degradation of behaviour (Kupfer et al 1987) in line with non-oxide superconductors. The oxides are somewhat more sensitive as a function of displacements per atom (Geerk et al 1987).

Several Japanese workers have studied the effects of fast neutron irradiation on both YBCO and the La compound (Atobe and Yoshida 1987, Atobe et al 1988, Yoshida and Atobe 1988), with results suggesting that very large critical temperatures can be achieved. These have not been verified. Contrary to the findings of Umezawa $e t$ al, recent work (Okada and Kuwakada 1989) indicates that one can achieve improved critical currents by neutron irradiation both at ambient reactor temperatures (360 K approximately) and at low temperatures $(20 \mathrm{~K})$, the critical temperature is degraded by irradiation at the reactor temperature, and improved somewhat by irradiation at $20 \mathrm{~K}$.

The critical temperature of $\mathrm{Pb}$-doped $\mathrm{BISCCO}$ has been found to fall with neutron irradiation (Herr et al 1989). The value of $T_{\mathrm{c}}$ measured after irradiation of $\mathrm{Pb}$-doped $\mathrm{BiSrCaCuO}$ superconductor with fast neutrons of energy $E>0.1 \mathrm{MeV}$ shows relatively little change for fluences up to $4.0 \times 10^{17}$ neutrons $\mathrm{cm}^{-2}$, but large changes (a reduction to $92.5 \mathrm{~K}$ from $102 \mathrm{~K}$ ) for fluences greater than $6.0 \times 10^{17} \mathrm{~cm}^{-2}$. There is thus a more sensitive dependence of fractional critical temperature with neutron fluence for BISCCO than for the lanthanum compound or YBCO, but this may be partly due to the absence of thermal neutron shielding during irradiation. Electrical resistance measurments strongly suggest the recovery of radiation-induced damage in BISCCO during room temperature storage, but certainly more experiments are necessary.

6.4.5. Ion beams. In line with non-superconducting oxides (White et al 1989) there are reports of new phases being formed on ion beam irradiation (Xiong et al 1988), as well as the expected features like the generation of dislocation loops and changes in critical temperature and on the conductivity at room temperature.

At temperatures above the critical temperature, the resistivity and Hall coefficient of YBCO behave like conventional metals for low ion-beam fluences, but shows a metal-insulator transition, apparently from a fall in carrier mobility rather than concentration (Valles et al 1989).

Groult et al (1988) have shown that the critical temperature of the surface superconducting form of $\mathrm{La}_{2} \mathrm{CuO}_{4}$, which exhibits semiconducting behaviour above its onset temperature, may be improved by heavy-ion irradiation (in this case $2.9 \mathrm{GeV}$ krypton ions). The actual nature of the defects induced by the irradiation is not well established, but it is conjectured that electronic stopping plays a major role in such an improvement of the superconducting properties.

In studies of the effects of oxygen and arsenic ion implantation into thin films of YBCO (Clark et al 1987b), the deposited energy needed to change the critical temperature was found to be $0.2 \mathrm{eV}$ per atom, while $1-2 \mathrm{eV}$ per atom was needed to affect the room temperature conductivity, $4 \mathrm{eV}$ per atom was needed to render the film amorphous. Similar changes in critical temperature were noted by Xiong et al (Xiong et al 1988) by irradiation of YBCO films at $77 \mathrm{~K}$. Egner et al (Egner et al 
1987) also irradiated YBCO with ion beams, in this case $\mathrm{HI}, \mathrm{He}$, and $\mathrm{Ar}$ ions at $293 \mathrm{~K}$ and $77 \mathrm{~K}$. The orthorhombic to tetragonal phase transformation resulted at between 0.01 and 0.03 displacements per atom (dpa). The transformation into the amorphous phase was observed at about 0.1 dpa after He irradiation at $77 \mathrm{~K}$.

Even at doses below those causing the orthorhombic to tetragonal transition, radiation-induced damage (dislocation loops) can be seen by in situ electron microscopy in EuBCO and GdBCO (Ruault et al 1988).

Oxygen and arsenic ions implanted (Clark et al 1988) to investigate the effects of ion irradiation on the superconducting, electrical and microstructural properties of thin films of $\mathrm{YBCO}$ show differing effects on grain boundaries and bulk properties. Superconducting transitions were found to degrade in polycrystalline films by a decoupling of the superconducting grains, while epitaxial films degrade due to bulk damage. The normalised conductivity, plotted as a function of the energy, expressed in dpa, which the incident ions deposit into elastic collisions with the target atoms shows that the curves for the oxygen and arsenic damage are effectively superimposed on each other. This means that the damage mechanism in the films is one of nuclear displacements (i.e. elastic processes) rather than electronic induced damage. This may limit processing in which the superconductor is exposed to a plasma, since then the ion bombardment is unshielded.

The effect of ion beams on critical current has received much attention since pinning is hoped to improve performance. Willis et al (1988a) irradiated sintered samples of EuBCO and $\mathrm{GdBCO}$ (both with about seven oxygens per formula unit) at $90^{\circ} \mathrm{C}$ with $800 \mathrm{MeV}$ protons, looking for the dependence on fluence of the critical current. There are two critical currents to consider: one is representative of intragranular conduction, and should be similar to results for single crystals; the other is intergranular conduction, presumably dominated by the Josephson weak links. The quantity measured in magnetization experiments is characteristic of intragranular conduction. Willis found the critical temperature to be depressed and the critical current enhanced. The size of the Meissner susceptibility, characterizing the flux expulsion properties of a superconductor, shows a strong systematic decrease with fluence for both Gd and Eu based compounds. The Meissner susceptibility decreases by a factor of about three between unirradiated and highly-irradiated samples. A small Meissner effect means that less flux is being expelled, which in turn implies stronger flux pinning in the irradiated sample. This should be reflected in an increased hysteresis in $M$ versus $H$ curves, and therefore in an increased critical magnetization current density. This was apparent in the results of Willis et al, and is in line with the increases noted for both YBCO and the La compound under neutron irradiation.

All these results are consistent with the general belief that defects act as additional pinning centres to those already present. If pinning is small, radiation damage tends to increase critical currents. However, if the critical current has been optimized, for example, by metallurgical means, it can only decrease by more complex and less discriminating mechanisms (Snead et al 1986). For YBCO, the critical current is known to decrease for fast neutron fluences greater than $2 \times 10^{18} \mathrm{n} \mathrm{cm}^{-2}(E>$ $0.1 \mathrm{MeV}$ ), at which point the critical temperature has been depressed by $4 \mathrm{~K}$, twice as far as in the proton irradiation experiments of Willis. The data and other results for GdBCO suggest that the critical current may be approaching a maximum value, as for neutron irradiation (Willis et al 1988b).

Clearly, there will be some cases where one expects the implanted species to play a chemical role. This has been noted (Kato et al 1988) for hydrogen ion implantation 
in YBCO. The observed shrinkage in the lattice and the fall shift of onset transition temperature suggested that the implanted protons deoxidized the YBCO compound. However, oxygen is NOT selectively removed from the chain sites.

Whereas most ablation results use lasers, there are reports of very fast ion-beam milling of YBCO (Nishi et al 1988) and of reactive ion beam etching (RIBE) using chlorine gas (Matsui et al 1988) useful for microfabrication of YBCO superconductors. These techniques should prove of use in mícrofabrication technology for superconductor applications to microelectronic devices.

\section{Summary}

In this review, we have surveyed those aspects of oxide superconductors which relate them most closely to conventional ceramic oxides. There are significant similarities, as well as important differences. Some of the methods and ideas that are valid for modelling ionic systems can be exploited for superconducting oxides. Through quantitative modelling there is potential to control defect processes and microstructure so as to enhance performance, and we have noted work that sheds light on this area. In particular, there are experiments that show enhanced critical currents, higher critical temperatures, greater resistance to atmospheric attack or greater mechanical strength, and parallels with other oxide systems may aid further gains (see the opening paragraphs of sections 3 and 4 , and subsections 4.2.1, 4.2.2, 4.2.3, 6.4.4 and 6.4.5).

Results reported have implications for preparation routes, for example the assymmetry between oxygen removal and addition. Low-temperature processing routes, such as irradiation or certain laser methods which allow the superconducting properties of a material to be changed locally, may have special advantages, for there is always a compromise between the stability of a desired phase (which may demand a low temperature) and the processes of manufacture, which-if purely thermal-would need a high temperature to proceed at a useful rate. It may be that special compounds or structures can be created by special methods to avoid processing temperatures above the stability limit of some useful metastable oxide.

There has been much theoretical work done on the superconducting oxides and several striking achievements. Ionic crystal calculations for YBCO based on the shell model, using empirical two-body interatomic potentials, can predict well the effects of doping with non-transition and transition metals ions at copper sites, and also the preferential substitutional sites for divalent and trivalent impurity ions and anions. Theoretical calculations of perfect lattice and defect properties of $\mathrm{M}_{2} \mathrm{CuO}_{4}$ (where $\mathrm{M} \equiv$ $\mathrm{La}, \mathrm{Pr}, \mathrm{Nd}, \mathrm{Al}$ ) based on interatomic potentials, largely from electron-gas calculations, can correctly predict the observed crystal structures for all these systems. This is quite a feat, since there are several alternative structures very close in energy. The predicted phonon density of states based on these simple electron-gas potentials proves to be a useful investigative fingerprint in the assessment of potential superconducting oxides.

There still remain both experimental and theoretical problems with the superconducting oxides. As for most oxides, in handling the superconducting oxides, we encounter the experimental problems of laboratory to laboratory differences and possibly significant inhomogeneities in single samples. These problems have been reduced somewhat by improved experimental techniques, but can make comparisons difficult. Experimental problems are more severe with YBCO than for BISCCO, since YBCO is prone to atmospheric attack, tends to react with substrates and exhibits compositional instability. 
At present, diffusion data for the oxide superconductors does not form a coherent picture. There are gross differences from group to group and the problems have not yet been wholly resolved. The radiation response and temperature dependence of the resistance of the superconducting oxides, even above the critical temperature, is not yet understood and oxides of structure similar to the superconducting oxides need to be studied.

The understanding of the properties of superconducting oxides has increased greatly over the last few years and new ways to change their properties are constantly being found. Old problems are being overcome and with such a large amount of experimental and theoretical work being channelled into the study of these materials, the days of being able to fabricate samples with optimal compositional and microstructural properties for their purpose cannot be too far away.

\section{References}

Alig R C and Bloom S 1975 Phys. Rev. Lett. 351522

Allan N L and Mackrodt W C 1988a High Temperature Superconductors (Mater. Res. Soc. Symp. Proc. 99) ed M B Brodsky and R C Dynes (Pittsburgh, Pa: Materials Research Society) p 797 1988b Ceramic Superconductors II ed M F Yan (American Ceramic Society) p 115 $1989 \mathrm{~J}$. Chem Soc. Faraday Trans. II 85385

Arizmendi L, Cabrera J M and Agullo-Lopez F 1984 Proc. Coll. on Basic Properties of Binary Oxides (La Rabida, Spain, 1983) (Seville: University of Seville Press) p 343

Atobe $\mathrm{K}$ and Yoshida H 1987 Phys. Rev. B 367194

Atobe K, Yoshida H, Okada M and Nakagawa M 1988 J. Nucl. Sci. Techrol. 25410

Auciello O, Athavale S, Hankins O E, Sito M, Schreiner A F and Biunno N 1988b Appl. Phys. Lett. 5372

Auciello O, Krauss A R, Santiago-Aviles J, Schreiner A F and Gruen D M 1988a Appl. Phys. Lett. 52239

Auvray-Gely M H 1989 Orsay Report CEA-R-5480

Babakishvili G O, Kekelidze G P, Kekelidze N P and Tsintsadze G A 1989 Fiz. Nizk. Temp. 141294

Babcock S E, Kelly T F, Lee P J, Seuntjens J M, Lavanier L A and Larbalestier D C 1988 Physica C 15225

Baetzold R C 1988 Phys. Rev. B 3811304

Bakker H, Welch D O and Lazareth O W 1987 Solid State Commun. 64237

Baldha G J, Jotania R B, Joshi H H, Pandya H N and Kulkarni R G 1989 Solid State Commun. 71 839

Becker C H and Pallix J B 1988 J. Appl. Phys. 64 5152

Bohandy J, Suter J, Kim B F, Moorjani K, Adrian F J 1987 Appl. Phys. Lett. 512161

Boiko B B, Korshunov F P, Gatalskii GV, Akimov A I, Gatalskaya V I, Demyanov S E and Stribuk E K 1988 Phys. Status Solidi a 107 K139

Bokhimi, Garcia-Ruiz A, Perez L, Orozco E, Asomoza R and Asomoza M 1989 Physica C 159654

Bridges F A, Davies G, Robertson J and Stoneham A M 1990 J. Phys.: Condens. Matter 22875

Burger J P, Lesueur L, Nicolas M, Da.ou J N, Dumoulin L and Vajda P 1987 J. Physique 481419

Bursill L A and Fan X D 1988 Phys. Status Solidi a 107503

Buttrey D J, Ganguly P, Honig J M, Rao C N R, Schartman R R and Subbanna G N 1988 J. Solid State Chem. 74253

Byme A S, Stickle W F, Yang C Y, Asano T and Rahman M M 1989 High $T_{c}$ Superconducting Thin Films, Devices and Applications (Proc. AIP Conf. Proc. 182) (Ridge, NY: American Institute of Physics) p 352

Catlow C R A and Mackrodt W C 1982 Computer Simulation of Solids (Berlin: Springer)

Catlow C R A and Stoneham A M 1983 J. Phys. C: Solid State Phys. 164321

Catlow C R A, Tomlinson S M, Islam M S and Leslie M 1988 J. Phys. C: Solid State Phys. 21 L1085

Cerezo A 1989 J. Phys.: Conders. Matter 1 SB 101

Chandrachood M R, Mulla I S, Gorwadka S M and Sinha A P B 1990 Appl. Phys. Lett. 56183

Chang C-A and Tsai J A 1988 Appl. Phys. Lett. 531976 
Chang C-A, Tsai J A and Farrell C E 1989 High $T_{c}$ Superconducting Thin Films, Devices and Applications (Proc. AIP Conf. Proc. 182) (Ridge, NY: American Institute of Physics) p 384

Chaudhari S M, Viswanathan R, Bendre S T, Nawale P P, Kanetlar S M and Ogale S B $1989 \mathrm{~J}$. Appl. Phys. 664509

Chen C H, Werder D J, Scheenmeyer L F, Gallagher P K and Waszczak J V 1988 Phys. Rev. B 38 2888

Chengyu $W$, Min C, Guoxing $X$ and Weimin Z 1987 Def, Diff. Data 53-54 483

Chiang Y-M, Rudman D A, Leung D K, Dkeda J A S, Roshko A and Fabes B D 1988 Physica C 152 77

Choisnet J, Bassat J M, Pilliere H, Odier P and Leblane M 1983 Solid State Commun, 661245

Choy T C and Stoneham A M $1990 \mathrm{~J}$. Phys.: Condens. Matter 2 939, 2867.

Chung G and Barr L W 1988 Solid State Commun. 67123

Clark G J, LeGoues F K, Marwick A D, Laibowitz R B and Koch R 1987a Appl. Phys. Lett. 511462

1988 Mater. Res. Soc. Symp. Proc. 99 p 127

Clark G J, Marwick A D, Kocli R H and Laibowitz R B 1987b Appl. Phys. Lett. 51139

Cohen L, Gray I R, Porch A and Waldram J R 1987 J. Phys. F: Met. Phys. 17 L179

Collins R T, Schlesinger Z, Chandrasekhar G V and Schafer M W 1989 Phys. Rev. B 392251

Cota L et al $1988 \mathrm{~J}$. Mater. Res. 3417

Couach $M$ and Khoder A F 1988 Phys. Rev. B 38748

Crawford M K, Farneth W E, Bordia R K and McCarron E M III 1988 Phys. Rev. B 373371

Cywinski R, Kilcoyne S H and Lomer J N 1988 Solid State Commun. 67 355

deGroot F M F, Grioni M, Fuggle J C, Ghijsen J, Sawatzky G A and Petersen H 1989 Phys. Rev. B 405715

Deshmukh S, Rothe E W, Reck G P, Kushida T and Xu Z G 1988 Appl. Phys. Lett. 532698

Dufour $L C$ and Nowotny J 1989 Oxide Surfaces (Amsterdam: Elsevier)

Egner B, Geerk J, Li H C, Linker G, Meyer O and Strehlau B 1987 Japan. J. Appl. Phys. I 26 Suppl. 26-3 (Proc. 18th Int. Conf. on Low Temperature Physics (Kyoto, Japan, 1987))

Etemad S, Aspnes D E, Kelly M K, Thompson R, Tarascon J-M and Hull G W 1988 Phys. Rev, B 373396

Farlow G C, White C W, McHargue C J and Appleton B R 1984 Mater. Res. Soc. Symp. Proc. 27 395

Farneth W E 1988 Mater. Res. Soc. Symp. Proc. 99977

Felner I, Nowik I and Yeshurun Y 1987 Phys. Rev. B 363923

Fiory A T and Grader G S 1988 Phys. Rev. B 389198

Flavell W R and Egdell R G 1988 Supercond. Sci. Technol. 1118

Fogarassy E, Fuchs C, Siffert P, Perriere J, Wang X Z and Rochet F 1988 Solid State Commun. 67 975

Freund F, King D, Knoble R and Kathrein H 1984 Adv. Cerem. 10119

Garriga M, Humilicek J, Cardona M and Schonherr E 1988 Solid State Commun. 661231

Geerk J, Li H C, Linker G, Meyer O, Politis C, Ratzel F, Smithey R, Strehlau B and Xi X X 1987 INFP KfH.Nachr. 19155

Ghosh A and Chakravorty D 1990 J. Phys.: Condens. Matter 2649

Ginder J M, Roe M G, Song Y, McCall R P, Gaines J R and Ehrenfreund E 1988 Phys. Rev. B 37 7506

Ginley D S, Nigrey P J, Venturini E L, Morosin B and Kwak F J 1987 Phys. Rev, B 36829

Glowacki B A, Highmore R J, Peters K F, Greer A L and Evetts J E 1988 Supercond. Sci. Technol. 17

Goldfarb R B, Clark A F, Braginski A I and Panson A J 1987 Cryogenics 27475

Goldschmidt V M, Barth $\mathrm{T}$, Lunde G and Zachariasen W H 1926 Skr. Norske Vidensk. Akad. 1, Mat.-Nat. 12

Golovashkin A I, Ivanenko O M, Leitus G I, Mitsen K V, Karpinski O G and Shamrai V F 1988 JETP Lett 46410

Goodenough J B 1987 Mater. Ed. 9623

1990 Supercond. Sci. Technol. 326

Grader G S, Gallagher P K, Thomson J and Gurvitch M 1988 Appl. Phys. Lett. A 45179

Groult D, Provost J, Raveau B, Studer F, Bouffard S, Jousett J C, Lewandowski S J, Toulemonde M and Rullier-Albenque F 1989 Europhys. Lett, 6151

Gupta A and Koren G 1988 Appl. Phys. Lett. 52665 
Hagen C W and Griessen R P 1989 Phys. Rev. Lett. 622857

Hakwraku Y, Sunsiyoshi F and Ogushe T 1988 Appl. Phys. Lett. 521528

Hangyo M, Nakashima S, Mizoguchi K, Fujii A, Mitsuishi A and Yotsuya $T 1988$ Solid State Commun. 65835

Harris L B and Nyang F K 1988 Solid State Commun. 67359

Hayes W and Stoneham A M 1985 Defects and Defect Processes in Non-metallic Solids (New York: Wiley-Interscience)

Healy P C, Myhra S and Stewart A M 1988 Phil. Mag. B 58257

Hegde M S 1988 Mater. Res. Bull, 231171

Hegde M S and Ganguly P 1988 Phys. Rev. B 384557

Henderson B 1988 Crit. Rev. Solid State Science 91

Herr Y-H, Lee K-H, Kim C-J, Lee H-G, Kim C-T, Hong G-W and Won D-Y 1989 Japan. J. Appl. Phys. 28 L1561

Hervieu M, Domenyes B and Michel C 1987 Europhys. Lett. 4205

Highmore R J, Evetts J E, Greer A L and Somekh R E 1987 Appl. Phys. Lett. 59566

Hill D M, Meyer III H M, Weaver J H, Gallo C F and Goretta K C 1988 Phys. Rev. B 3811331

Hoare J P, Masri P and Tasker P W 1983 AERE Report R10752

Hodgson E R and Agullo-Lopez F $1989 \mathrm{~J}$. Phys.: Condens. Matter 110015

Hofmann A, Kronmuller H, Moser N, Reisser R, Schule P and Dworscliak F 1988 Physica C 156528

Horowitz H S, Bordia R K, Flippen R B, Johnson R E and Chowdry U 1988 Mater. Res. Bull, 23 821

Humphreys R G, Satchell J S, Chew N G and Edwards J A 1989 Appl. Phys. Lett. 5475

Ikuma Y and Akiyoshi S $1988 \mathrm{~J}$. Appl. Phys. 643915

Islam M S and Baetzold R C 1989 Phys. Rev. B 4010926

Islam M S, Leslie M, Tomlinson S M and Catlow C R A 1988 J. Phys. C: Solid State Phys. 21 L109 J. Chem. Soc. Faraday Trans. 1989 II 85 (special issue on the Mott-Littleton method)

Jorgensen J D, Dabrowski B, Pei S, Richards D R and Hinks D G 1989 Phys. Rev. B 402187

Kaiser D L, Gayle F W, Rath R S and Schwarzendruber L J 1989 J. Mater. Res. 4745

Kato T, Usami K, Kuniya J and Matsuda S-P 1988 Japan. J. Appl. Phys. 27 L1104

Kelly M K, Barboux P, Tarascon J-M, Aspnes D E, Bonnor W A and Mortís P A 1988 Phys. Rev. B 38870

Kent A D, Maggio-Aprile I, Niedermann $P$ and Fischer $O 1989$ Phys. Rev, B 3912363

Khachaturyan A G and Morris J W 1988 Phys. Rev. Lett. 61215

1990 Phys. Rev. Lett. 6476

Kim Y H, Foster C M, Heeger A J, Cox S and Stucky G 1988 Phys. Rev. B 386478

Kingery W D, Bowen H K and Uhlmann D R 1976 Introduction to Ceramics 2nd edn (New York: Wiley)

Kirk M D, Eom C B, Oh B, Spielman S R, Beasley M R, Kapitulnik A, Geballe T H and Quate C F 1988a Appl. Phys. Lett. 522071

Kirk M D, Nogami J, Baski A A, Mitzi D B, Kapitulnik A, Geballe T H and Quate C F 1988b Science 2421673

Kistenmacher T J 1988 Phys. Rev. B 388862

Kogure T, Zhang Y, Levonmaa R, Kontra R, Wang W-X, Rudman D A, Yurek G J and Vander Sande J B 1988 Physica C 156707

Koinuma H, Fukuda K, Hashimoto $T$ and Fuezi K 1988 Japan. J. Appl. Phys. 27 L1216

Kolinsky P V, Mary P, Harrison M R, Miller P and Jedamzik D 1989 Superçond. Sci. Technol. 1333

Kourouklis G A, Jayaraman A, Batlogg B, Cava R J and Stavola M 1987 Phys. Rev. 368320

Krebs H U and Wordenweber R $1988 \mathrm{~J}$. Appl. Phys. 631642

Kroeger D M, Choudhury A, Brynestad J, Williams R K, Padgett R A and Coghlan W A 1988 J. Appl. Phys. 64331

Krol D M, Stavola M, Weber W, Schneemeyer L F, Waszczak J V, Zahurak S M and Kosinski S G 1988 Phys. Rev. B $\mathbf{3 6} 8325$

Kuiper P, Kruizinga G, Ghijsen J, Sawatzky G A and Verwiej H 1989 Phys. Rev. Lett. 62221

Kullmer R and Bauerle D 1988 Appl. Phys. A 47103

Kupfer H, Apfelstedt I, Schauer W, Flukiger R, Meier-Hirmer R, Wuhl H and Scheurer H $1987 Z$. Phys. B 69167

Kuroda K, Kojima K, Tanioku M, Yokoyama K and Hamanaka K 1989 Japan. J. Appl. Phys. 28 1586 
LaGraff J R, Behrman E C, Taylor J A T, Rotella F J, Jorgensen J D, Wang L Q and Mattocks P G 1989 Phys. Rev. B 39347

Lewis G V 1983 DPhil Thesis University College, London

Lewis $\mathrm{G}$ and Catlow C R A 1983 Radiat. Eff. Def. Solids 73307

Li J, Wang S Qt Mayer J W and Tu K N 1989a Phys. Rev. B 3912367

Li Q, Weschenfelder F, Meyer O, Li X X, Linker G and Geerk J 1989b J. Less-Common Met. 89295

Liang Y H, Chen J and Feng D 1989 Appl. Phys. Lett, 54368

Liberts G, Eyett M and Bauerle D 1988a Appl. Phys. A 45313 1988bAppl. Phys. A 46331

Liu Y, Pangilinan G, Sooryakumar R, Sumner M J and Lemberger T R 1988 J. Appl. Phys, 64 3598

Lopez-Morales M E, Rios-Jara D, Taguena J, Escudero R, La Placa S, Lee V Y, Engler E M and Grant P M 1990 Phys. Rev. B in press

Loquet J-P, Vanacken J, Wuyts B, Bruynseraede Y, Zhang K and Schuller I K 1988 Europhys. Lett. 7469

Luschik C B, Kuusmann I L, Feldbakh E K, Liblik P K, Savikhina T I and Meriloo I A 1987 Sov. Phys.-Solid State 292097

Ma Q Y, Licata T J, Wu X and Yang E S 1988 Appl. Phys. Lett. 532229

Mackrodt W C $1989 \mathrm{~J}$. Chem Soc. Faraday Trans. II 85541

Maeda A, Yabe T, Takebayashi S, Hase M and Uchinokura K 1990 Phys. Rev. B 414112

Mannhart J, Scheuermann M, Tsuei C C, Oprysko M M, Chi C C, Umbach C P Koch R H and Miller C 1988 Appl. Phys. Lett. 521271

Manthiram A, Tang X X and Goodenough J B 1988 Phys. Rev. B 373734

Marwick A D, Guarnieri C R and Manoyan J M 1988 Appl. Phys. Lett. 532713

Masumi T, Shimada H and Minami H 1987 J. Phys. Soc. Japan 563009

Matsui S, Takado N, Tsuge H and Asakawa K 1988 Appl. Phys. Lett, 5269

McCarty K F, Hamilton J C, Shelton R N and Ginley D S 1988 Phys. Rev. B 382914

Miller J H, Holder S L and Hunn J D 1989 Appl. Phys. Lett. 542256

Monod P, Ribault M, D'Yvoire F, Jegoudez J, Collin G and Revcolevschi A 1987 J. Physique 48369

Morss L R, Sonnenberger D C and Thorn R J 1988 inorg. Chem. 272106

Moseley P T and Tofield B C (ed.) 1987 Solid State Gas Sensors (Bristol: Adam Hilger)

J. Chem Soc. Faraday Trans. II 198985 (special issue on the Mott-Littleton method)

Mueller F M, Chen S P, Prueitt M L, Smith J F, Smith J L and Wohlleben D 1988 Phys. Rev. B 37 5837

Muraleedharan K, Tomy C V, Malik S K, Prasad R and Soni N C 1988 Solid State Commun. 68227

Naguib H M and Kelly R 1975 Radiat. Eff. 251

Naito $M$ et al 1987 Phys. Rev. B 357228

Nakajima H, Yamaguchi S, Iwasaki K, Morita H and Fujimori H 1988 Appl. Phys. Lett. 531437

Nakamura K and Ogawa K 1988 Japan. J. Appl. Phys. 27577

Nastasi M, Parkin D M, Zocco T G, Koike J and Okamoto P R 1988 Appl. Phys. Lett. 531326

Ni B, Munakata T, Matsushita T, Iwakuma M, Funaki K, Takeo M and Yamafuji K 1988 Japan. J. Appl. Phys. 271658

Nishi Y, Moriya S, Inoue N, Tokunaga S and Shima T 1988 J. Mater, Sci. Lett. 7281

Oates W A and Stoneham A M 1983 J. Phys. F: Met. Phys. 132427

Oesterreicher H and Smith M 1987 Mater. Res. Bull. 221709

Okada M and Kawakubo T 1989 Radiat. Eff. Def. Solids 108137

O'Sullivan E J M and Chang B P 1988 Appl. Phys. Lett. 521441

Palhan L, Brokman A, Felner I, Brettschneider M, Yacoby Y and Weger M 1988 Solid State Commun. 68313

Park J-H, Kostic P and Singh J P 1988 Mater. Lett. 6393

Park Y K, Lee H K, Hwang N M, Kwon H C, Park J C and Yoon D N 1988 J. Am. Ceram. Soc. 71 $\mathrm{C} 297$

Pei S, Jorgensen J D, Dabrowski B, Hinks D G, Richards D R and Mitchell A W 1990 Phys. Rev. B 414126

Peterson G G, Weinberger B R, Lynds L and Krasinski H A 1988 J. Mater. Res. 3605

Polman E A, Fransen T and Gellings P J 1989 J. Phys.: Condens. Matter 14497

Poole C P, Datta $T$ and Farach H 1989 Copper Oxide Superconductors (New York: Wiley)

Qiu S L, Ruckman M W, Brookes N B, Johnson P D, Chen J, Lin C L, Strongin M, Sinkovis B, Crow $\mathrm{J}$ E and Jee C-S 1988 Phys. Rev. B 373747 
Ramesh R, Wang E, Greene L H, Hegde M S and Tarascon J-M 1990 J. Mater. Res. 5251

Rao C N R, Ganguly P, Gopalakrishnan J and Sharma D D 1987 Mater. Res. Bull, 221159

Regnier P, Gupta R P and Truchot P 1988 J. Phys. C: Solid State Phys. 21 L463

Rigney M M, Poole C P and Farach H A 1989 J. Phys. Chem. Soc. 509

Rosen H, Engler E M, Stranf T C, Lee V Y and Bethune D 1987 Phys. Rev. B 36726

Rosenberg R A and Wen C-R 1988 Phys. Rev. B 379852

Roth M, Halperin A and Katz S 1988 Solid State Commun. 67105

Routbort J L, Rothman S J, Flandermeyer B K, Nowicki L J and Baker J E 1988 J. Mater. Res. 3 116

Ruault M-O, Bernas H, Lesucur J, Dumoulin L, Nicolas M, Burger J-P, Gasgnier M, Noel H, Gougeon P, Polet M and Levet J C 1988 Europhys. Lett. 7435

Ruckman M W, Heald S M, Di Marzio D, Chen H, Moodenbaugh A. and Yang C Y 1989 High $T_{c}$ Superconducting Thin Films, Devices and Applications (Proc. AIP Conf. Proc. 182) (Ridge, NY: American Institute of Physics) p 360

Saenger K 1989 J. Appl. Phys. 664435

Saiz E and Moya J S 1988 Mat. Lett. 6369

Salomons E, Koeman N, Brouwer R, de Groot D G and Griesses R 1987 Solid State Commun. 64 1141

Sambe H, Ramaker D, Parenteau L and Sanoh L 1987 Phys. Rev. Lett. 59236

Schirmer O and Muller K A 1973 Phys. Rev. B 72986

Schrott A G, Singco G and Tu K N 1989 Appl. Phys. Lett. 552126

Schuller I K, Hinks D G, Beno M A, Capone II D W, Soderholm L, Loquet J-P, Bruynseraede Y, Segre $C U$ and Zhang K 1987 Solid State Commun. 63385

Shaked H, Veal B W, Faber J, Hitterman R L, Balachandran U, Tomlins G, Shi H, Morss L and Paulikas A P 1990 Phys. Rev. B 414173

Shannon R D and Prewitt C T 1969 Acta Crystallogr. B 25925

Shi D, Krucpzak J, Tang M, Chen N and Bhadra R 1989 J. Appl. Phys. 664325

Singh J P, Leu H J, Poeppel R B, Van Voorhees E, Goudey G T, Winsley K and Shi D 1989a J. Appl. Phys. 663154

Singh P, Nyayate M, Devare S H and Devare H G 1989b Phys. Rev. B 392308

Smith D A, Chisholm M F and Clabes J 1988 Appl. Phys. Lett. 532344

Snead J C L and Luhman T 1986 Physics of Radiation Effects in Crystals (Amsterdam: Elsevier) ch 6

Song Y, Chen D and Gaines J R 1990 J. Mater. Res. 527

Stankevich V G, Svechnikov N Yu, Kaznacheev K V, Kink R A, Niedrais Kh E, Golubev V N, Kosyev V Ya, Simirsku Yu N and Tsetlin M B 1988 JETP Lett. 47385

Steiner P, Courths R, Kinsinger V, Sander I, Siegwart B and Hufner $S 1987$ Appl. Phys, A 4475

Steiner $\mathrm{P}$ et al 1988 Z. Phys. B 69449

Stockbauer R L, Robey S W, Kurtz R L, Mueller D, Shih A, Singh A K, Toth L and Osofsky M 1989 High $T_{c}$ Superconducting Thin Films, Devices and Applications (Proc. AIP Conf. Proc. 182) (Ridge, NY: American Institute of Physics) p 276

Stoneharn A M 1987 AERE Report M-3639

- 1989a J. Chem Soc. Faraday Trans. II 85505

1989b Phys. Scr. 2517

- 1989c Nucl. Instrum. Methods B 48389

Stoneham A M and Durham P J $1973 \mathrm{~J}$. Phys. Chem. Solids 342127

Stoneham A M S and Sangster M J L 1983 Radiat. Eff. Def. Solids 73267

Stoneham A M and Tasker P W 1989 Oxide Surfaces ed L C Dufour and I Nowotny (Amsterdam: Elsevier) p 1

Strauven H, Loquet J P, Verbeke O B and Bruynerseraede Y 1988 Solid State Commun. 65293

Strobel P, Capponi J J, Marezio M and Monod P 1987 Solid State Commun. 64513

Su M-Y, Dorris S E and Mason T O $1988 J$. Solid State Chem. 75381

Sugita T, Yabuuchi M, Murase K, Okabayashi H, Gamo K and Namba S 1988 Solid State Commun. 6795

Summers G P, Burke E A, Chrisey D B, Nastasi M and Tesmer J 1989 Appl. Phys. Lett. 551469

Tanaka M, Takahashi T, Katayama-Yoshida $H_{1}$ Yarnazaki S, Fujinarni M, Okabe Y, Mizutani W, Ono M and Kajimura K 1989 Nature 339691

Tanimura K, Tanaka T and Itoh N 1984 Nucl. Instrum. Methods B 1187 
Tao Y K, Swinnea J S, Manthiram A, Kim J S, Goodenough J B and Steinfink H 1988 High Temperature Superconductors (Mater. Res. Soc. Symp. Proc, 99) ed M B Brodsky and R. Dynes (Pittsburgh, Pa: Materials Research Society) p 519

Tarascon J M, Mckinnon W R, Greene L H, Hull GW and Vogel E M 1987 Phys. Rev. B 36226

Tasker P W 1988 Solid State Ion. 8233

Tasker P W and Duffy D 1983 Phil. Mag. A 47817

Tasker P W and Dufour L C 1988 Mater. Sci. Forum 29275

Tasker $\mathrm{P}$ W and Stoneham A M 1984 Br. Ceram. Proc, 341

Teodorescu V S, Nistor L C and Nistor S V 1989 J. Appl. Phys. 663682

Tissue B M and Wright J C $1988 \mathrm{~J}$. Lumin. 42173

Torrance J B and Metzger R M 1989 Phys. Rev. Lett. 631515

Toyozawa $Y 1981 J$. Phys. Soc. Japan 501861

Tu K N, Park S I and Tsuei C C 1987 Appl. Phys. Lett. 512158

Tu K N, Tsuei C C, Park S I and Levi A 1988 Phys, Rev, B 38772

Umezawa A, Crabtree G W, Liu J Z, Weber H W, Kwok W K, Nunez L H, Moran T J and Sowers C H 1987 Phys, Rev. B 367151

Vaidya S N, Srinivasan M P, Malik S K, Tomy C V and Adroja D T 1988 Solid State Commun. 67 119

Valles J M, White A E, Short K T, Dynes R C, Garno J P, Levi A F J, Anzlowar M and Baldwin K 1989 Phys. Rev. B 3911599

van de Leempt L E C, Van Bentum P J M, Schreurs L W M and Van Kempen H 1988 Physica C 15299

van Dover R B, Gyorgy E M, Schneemeyer L F, Mitchell J W, Rao K V Puzniak R and Waszczak J V 1989 Nature 34255

Vasek P, Smrcka L, Dominec J, Pesek M, Smrckova O and Sykorova D 1989 Solid State Commun. 6923

Verhoeven J D, Gibson E D, Chumbley L S, McCallum R W and Baker H H 1988 J. Appl. Phys. 64 761

von Stumberg A W, Che N and Goretta K C 1989 J. Appl. Phys. 662079

Wasa K, Adachi H, Hirochi K, Setsume K, Kamada T and Kitabatake M 1988 Thin Solid Films 163 175

Wells A F 1987 Structural Inorganic Chemistry (Oxford: Clarendon) p 1382

Wells B O, Lindberg P A P, Shen Z-X, Dessau D S, Lindau I, Spicer W E, Mitzi D B and Kapitulnik A 1989 High $T_{c}$ Superconducting Thin Films, Devices and Applications (Proc. AIP Conf. Proc. 182) (Ridge, NY: American Institute of Physics) p 391

Wen $S$, Song $X$ and Feng $J 1988$ Mater, Lett. 6385

Werfel F, Heinonen $M$ and Suoninen E $1988 Z$. Phys. 70317

White C W, McHargue C J, Sklad P S, Boatner L A and Farlow G C 1989 Mater, Sci. Rep. 441

Wills J O, Cooke D W, Brown R D, Cost J R, Smith J F, Smith J L and Aikin R M 1988a Appl. Phys. Lett. 53417

Willis $\mathrm{J} O$, Cost $\mathrm{J}$ R, Brown R D, Thompson J D and Peterson D E 1988b High Temperature Superconductors (Mater. Res. Soc. Symp. Proc. 99) ed M B Brodsky and R C Dynes (Pittsburgh, $\mathrm{Pa}$ : Matcrials Research Society) p 391

Wisniewski A, Baran M, Przyslupski P, Szymczak H and Pajaczkowska A 1988 Solid State Commun. 65577

Xiao G, Streitz F H, Cieplak M Z, Bakhshaj A, Gavrin A and Chien C L 1988 Phys. Rev. B 38776

Xie X M, Chen T G and Wu Z L 1989 Phys. Rev. B 404549

Xiong G C, Li H C, Linker G and Meyer O 1988 Phys. Rev. B 38240

Yamaguchi S, Terabe K, Imai A and Iguchi Y 1988 Japan. J. Appl. Phys. 27 L220

Yan M F, Ling H C, O'Bryan H M, Gallagher P K and Rhodes W W 1988 Mater. Sci. Eng. B I 119

Yoshida $\mathrm{H}$ and Atobe $\mathrm{K} 1988$ Physica $\mathrm{C} 156225$

Zandbcrgen H W, Gronsky R and Thomas G 1988 Phys. Status Solidi a 105207

Zhang J, Yang M and Chen T 1988 Mater. Lett. 6379

Zhang X, Catlow C R A and Zhou W 1990 Physica C 168417 